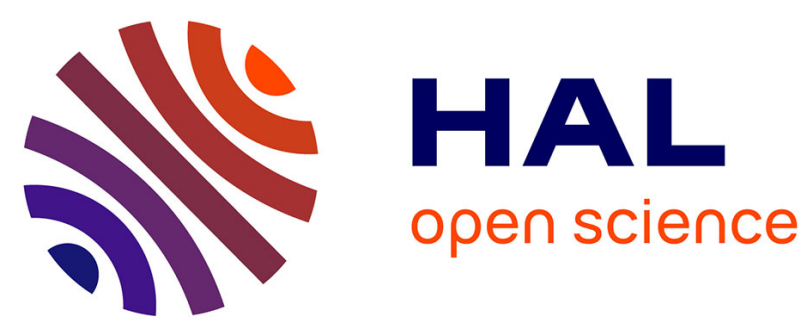

\title{
LOCALLY CONFORMALLY SYMPLECTIC STRUCTURES ON COMPACT NON- KAHLER COMPLEX SURFACES
}

Vestislav Apostolov, Georges Dloussky

\section{- To cite this version:}

Vestislav Apostolov, Georges Dloussky. LOCALLY CONFORMALLY SYMPLECTIC STRUCTURES ON COMPACT NON- KAHLER COMPLEX SURFACES. International Mathematics Research Notices, 2015, 10.1093/imrn/rnv211 . halshs-01108138v2

\section{HAL Id: halshs-01108138 \\ https://shs.hal.science/halshs-01108138v2}

Submitted on 10 Nov 2015

HAL is a multi-disciplinary open access archive for the deposit and dissemination of scientific research documents, whether they are published or not. The documents may come from teaching and research institutions in France or abroad, or from public or private research centers.
L'archive ouverte pluridisciplinaire HAL, est destinée au dépôt et à la diffusion de documents scientifiques de niveau recherche, publiés ou non, émanant des établissements d'enseignement et de recherche français ou étrangers, des laboratoires publics ou privés.

\section{()ㅜㅇㅜ}

Distributed under a Creative Commons Attribution - NoDerivatives| 4.0 International 


\section{Locally Conformally Symplectic Structures on Compact Non-Kähler Complex Surfaces}

\section{Vestislav Apostolov ${ }^{1}$ and Georges Dloussky ${ }^{2}$}

${ }^{1}$ Département de Mathématiques, UQAM, C.P. 8888, Succ. Centre-ville, Montréal, Québec H3C 3P8, Canada and ${ }^{2}$ Aix-Marseille University, CNRS, Centrale Marseille, I2M, UMR 7373, 13453, 39 rue F. Joliot-Curie 13411, Marseille Cedex 13, France

Correspondence to be sent to: e-mail: apostolov.vestislav@uqam.ca

We prove that every compact complex surface with odd first Betti number admits a locally conformally symplectic 2-form which tames the underlying almost complex structure.

\section{Introduction}

It is a well-known result $[11,30,42,46]$ that a compact complex surface $S=(M, J)$ admits a Kähler metric if and only if its first Betti number $b_{1}(M)$ is even. A cornerstone for the proof of this result is the fact, proved independently in [18, Lemme II.3; 21, p. 185] (see also [42, p. 143, Proposition 1.6] for the case of a K3 surface), that $b_{1}(M)$ is even if and only if $M$ admits a symplectic form $\omega$ which tames $J$, in the sense that the $(1,1)$ part of $\omega$ is positive-definite. This and the methods of proof in [11,30] inspired the so-called "tamed to compatible" conjecture in symplectic geometry, which asks whether an almost complex structure on $M$ which is tamed by a symplectic form admits a compatible symplectic form; see $[15,43]$.

A natural extension of the theory of Kähler manifolds to the non-Kählerian complex case can be obtained through the notion of locally conformally Kähler metrics, 
introduced and studied in foundational work by F. Tricerri and I. Vaisman; see for example, [14, 38] for an overview. Recall that a locally conformally Kähler (or lcK) metric on a complex manifold $X=(M, J)$ is defined by a positive-definite $(1,1)$-form $F$ satisfying $d F=\theta \wedge F$ for a closed 1 -form $\theta$. The 1 -form $\theta$ is uniquely determined and is referred to as the Lee form of $F$. The corresponding Hermitian metric $g(\cdot, \cdot)=F(\cdot, J \cdot)$ defines a conformal class $c$ on $M$. Changing the Hermitian metric $\tilde{g}=e^{f} g$ within $c$ amounts to transforming the Lee form by $\tilde{\theta}=\theta+d f$, so that the de Rham class $[\theta]$ is an invariant of the conformal class $c$.

Of particular interest is the case of compact complex surfaces, where recent works [6, 9, 10, 19] showed that lcK metric exists for all known examples of compact complex surfaces with odd first Betti number, with the exception of the complex surfaces obtained by blowing-up points of certain Inoue surfaces with zero second Betti number, described in [6]. However, a general existence result is still to come.

In this paper we study, on a compact complex surface $S=(M, J)$ with odd first Betti number, the problem of existence of locally conformally symplectic forms $\omega$ which tame $J$, that is, 2-forms $\omega$ satisfying $d \omega=\theta \wedge \omega$ for a closed 1-form $\theta$ (called Lee form of $\omega$ ), and such that the $(1,1)$-part of $\omega$ is positive-definite. This is, in general, a weaker condition than the existence of lcK metrics, which turns out to be related to the theory of bihermitian conformal structures $[2,40]$ in the case when the $\omega$-conjugate of $J$ determines another integrable almost-complex structure $J^{\omega}$ on $M$, see [1].

We establish the following general existence result, which we believe is an important step towards the resolution of the existence problems for both lcK and bihermitian conformal structures on a non-Kähler complex surface, and which answers in the positive (in the case of complex surfaces) a question raised in [38, Open Problem 1].

Theorem 1.1. Any compact complex surface $S=(M, J)$ with odd first Betti number admits a locally conformally symplectic form $\omega$ which tames $J$.

The above theorem is derived from another existence result concerning a conformal class of Hermitian metrics on $S=(M, J)$, which can be regarded as a twisted version of Gauduchon's celebrated theorem [17], and can be stated as follows. Let $a \in H_{d R}^{1}(M)$ be a de Rham cohomology class and $\alpha \in a$ a closed 1 -form in $a$. Denote by $d_{\alpha}:=d-\alpha \wedge$. the twisted differential operator defining the Lichnerowicz-Novikov complex, and let $d_{\alpha}^{c}:=J d J^{-1}$.

Theorem 1.2. Let $S=(M, J)$ be a compact complex surface with odd first Betti number, and $c$ a conformal class of Hermitian metrics on $S$. Then, there exists a non-zero de 
Rham class $a \in H_{d R}^{1}(M)$ such that for any metric $g \in c$, there exists a representative $\alpha \in a$ such that the fundamental 2-form $F$ of $g$ satisfies

$$
d_{\alpha} d_{\alpha}^{c} F=0 .
$$

The de Rham class $a$ appearing in Theorem 1.2 determines, via the exponential map

$$
H_{d R}^{1}(S, \mathbb{C}) \stackrel{\exp }{\hookrightarrow} H^{1}\left(S, \mathbb{C}^{*}\right) \longrightarrow \operatorname{Pic}_{0}(S),
$$

a flat holomorphic line bundle $\mathcal{L}_{a}$, and we derive Theorem 1.1 from Theorem 1.2 by showing that, when $H^{2}\left(S, \mathcal{L}_{a}\right)=\{0\}, S$ also admits a locally conformally symplectic form with a Lee form $\alpha$. The proofs of Theorems 1.1 and 1.2 are presented in Section 2, whereas the necessary analytical tools are collected in Appendix.

In the light of the "tamed to compatible" conjecture mentioned above, it is natural to compare the existence on $S$ of lcK metrics and of locally conformally symplectic forms taming $J$, through the corresponding de Rham classes of their Lee forms. We thus introduce in Section 3 the subset $\mathcal{C}(S)$ (respectively, $\mathcal{T}(S)$ ) in $H_{d R}^{1}(M)$ of classes $a$ for which there exists an lcK metric on $S$ with Lee form $\theta \in a$ (respectively, for which there exists a locally conformally symplectic form which tames $J$, with Lee form in a). We obviously have the inclusion $\mathcal{C}(S) \subseteq \mathcal{T}(S)$, and one may ask (see also [38; 9, Remark 9]).

Problem 1.3. Let $S=(M, J)$ be a compact complex surface with odd first Betti number. Determine the set $\mathcal{T}(S) \subset H_{d R}^{1}(M)$ of classes $a$ for which there exists a locally conformally symplectic form $\omega$ which tames $J$ and has a Lee form $\theta \in a$. Is $\mathcal{T}(S)$ strictly bigger than $\mathcal{C}(S)$ ?

Our initial motivation to study the above problem came from the theory of bihermitian conformal structures developed in [1], where the existence of the latter was reduced to answering the question of whether certain classes $a \in H_{d R}^{1}(M)$ belong to $\mathcal{T}(S)$ and $\mathcal{C}(S)$.

A number of partial results concerning Problem 1.3 are obtained in Section 4, where we specialize to the case of a compact complex surface with first Betti number equal to 1 .

In the last Section 5 of the paper, we consider some examples of non-Kähler complex surfaces in the Kodaira class VII (i.e. satisfying $H^{0}\left(S, \mathcal{K}_{S}^{\ell}\right)=\{0\}$ for all $\ell \geq 1$, where $\mathcal{K}_{S}$ stands for the canonical line bundle of $S$, see [5]), for which a complete answer to the above problem can be given. It is known that, in this case, the first Betti number equals 1 
(see e.g. [5]), and that the degree with respect to some Gauduchon metric on $S$ of the holomorphic line bundles determined via (1) induces an orientation on $H_{d R}^{1}(S) \cong(\mathbb{R},>)$, which turns out to be independent of the choice of a Gauduchon metric (see [45, Remark 2.4] or Lemma 4.1). Thus, for any compact complex surface in the Kodaira class VII, one can naturally identify $H_{d R}^{1}(S)$ with the oriented real line $(-\infty,+\infty)$. In this notation, a combination of Propositions 5.1 and 5.2 gives the following theorem.

Theorem 1.4. Let $S$ be a compact complex surface with a minimal model $S_{0}$.

(i) If $S_{0}$ is a Hopf surface, then $\mathcal{T}(S)=\mathcal{C}(S)=(-\infty, 0)$.

(ii) If $S$ is an Inoue surface of the type $S_{N, p, q, r ; u}^{+}$with $u \in \mathbb{C} \backslash \mathbb{R}$, then $\mathcal{C}(S)=\emptyset$ and $\mathcal{T}(S)=\left\{a_{0}\right\}$.

(iii) If $S$ is an Inoue surface of the type $S_{N, p, q, r ; u}^{+}$with $u \in \mathbb{R}$, then $\mathcal{C}(S)=\mathcal{T}(S)=\left\{a_{0}\right\}$, where $a_{0} \in H_{d R}^{1}(S)$ denotes the de Rham class for which the holomorphic line bundle determined by (1) is isomorphic to the anti-canonical line bundle $\mathcal{K}_{S}^{*}$.

\section{Existence of Locally Conformally Symplectic Forms Taming the Complex Structure}

Throughout the paper, we shall use the following convention.

Convention 2.1. Let $\alpha$ be a closed 1-form on $M$, representing a de Rham class $a=[\alpha]$. We denote by $L_{\alpha}$ the trivial real line bundle over $M$, endowed with a (non-trivial) flat connection $\nabla^{\alpha} s:=d s-\alpha \otimes s$, where $s$ is a smooth section of $L$. Similarly, $\nabla^{\alpha}$ induces a holomorphic structure on the complex bundle $\mathcal{L}_{\alpha}:=L_{\alpha} \otimes \mathbb{C}$. Writing $\alpha_{\mid U_{i}}=d f_{i}$ on an open covering $\mathfrak{U}=\left(U_{i}\right)$ of $M, s_{0}:=\left(U_{i}, e^{f_{i}}\right)$ defines a nowhere vanishing smooth parallel (respectively, holomorphic) section of $L_{\alpha}$ (respectively, $\mathcal{L}_{\alpha}$ ). In terms of (1), $\mathcal{L}_{\alpha}$ represents the isomorphism class of flat holomorphic line bundles determined by the deRham class $a=[\alpha]$. In what follows, we shall tacitly identify the real flat bundles $L_{\alpha}$ and $L_{\beta}$ (respectively, the flat holomorphic line bundles $\mathcal{L}_{\alpha}$ and $\mathcal{L}_{\beta}$ ) associated to different representatives $\alpha, \beta \in a$ and (slightly abusively) denote by $L_{a}$ (respectively, $\mathcal{L}_{a}$ ) either of them.

Let $L=L_{a}$ be the flat real line bundle over $M$ determined by $\alpha \in a, a \in H_{d R}^{1}(M)$ via the Convention 2.1, and denote by $L^{*}=L_{-a}$ its dual. The differential operator $d_{\alpha}=d-\alpha \wedge$. defines the Lichnerowicz-Novikov complex

$$
\cdots \stackrel{d_{\alpha}}{\rightarrow} \Omega^{k-1}(M) \stackrel{d_{\alpha}}{\rightarrow} \Omega^{k}(M) \stackrel{d_{\alpha}}{\rightarrow} \cdots
$$


which is isomorphic to the de Rham complex of differential forms with values in $L^{*}$

$$
\cdots \stackrel{d_{L^{*}}}{\rightarrow} \Omega^{k-1}\left(M, L^{*}\right) \stackrel{d_{L^{*}}}{\rightarrow} \Omega^{k}\left(M, L^{*}\right) \stackrel{d_{L^{*}}}{\rightarrow} \cdots
$$

In particular, we have an isomorphism between the cohomology groups

$$
H_{\alpha}^{k}(M) \simeq H_{d^{*}}^{k}\left(M, L^{*}\right)
$$

Considering the Dolbeault cohomology groups of $S$ with values in the flat holomorphic line bundle $\mathcal{L}^{*}$, we have

$$
d_{L^{*}}=\partial_{\mathcal{L}^{*}}+\bar{\partial}_{\mathcal{L}^{*}}, \quad \text { and } \quad d_{\alpha}=\partial_{\alpha}+\bar{\partial}_{\alpha}
$$

with

$$
\partial_{\alpha}=\partial-\alpha^{1,0} \wedge \quad \text { and } \quad \bar{\partial}_{\alpha}=\bar{\partial}-\alpha^{0,1} \wedge,
$$

giving rise to the isomorphisms

$$
H_{\bar{\partial}_{\alpha}}^{p, q}(S) \simeq H_{\bar{\partial}_{\mathcal{L}^{*}}^{p}}^{p, q}\left(S, \mathcal{L}^{*}\right)
$$

Definition 2.2. Let $X=(M, J)$ be a complex manifold. We shall say that a differentiable 2 -form $\omega$ is a locally conformally symplectic form taming $J$ if there exists a closed differentiable 1-form $\alpha$ such that $d_{\alpha} \omega=0$, and the $(1,1)$-part $\omega^{1,1}$ of $\omega$ is positive definite. If, furthermore, $\omega$ is of type $(1,1)$, it defines a locally conformally Kähler structure on $X$. The 1 -form $\alpha$ is called the Lee form $\alpha$ of $\omega$.

Remark 2.3. In terms of the isomorphism between (2) and (3), if we write $\alpha_{\mid U_{i}}=d f_{i}$ on an open covering $\mathfrak{U}=\left(U_{i}\right)$ of $M$, then $\omega_{i \mid U_{i}}:=e^{-f_{i}} \omega$ defines a $d_{L^{*}}$-closed 2-form with values in $L^{*}$, whose $(1,1)$-part is positive definite (for the latter we use the fact that $L^{*}=L_{-a}$ is defined by the co-cycle $\left(e^{f_{j}-f_{i}}, U_{i j}=U_{i} \cap U_{j}\right)$ which consists of positive constant real functions). Similarly, if $\omega$ is a locally conformally symplectic form taming $J$ with $d_{\alpha} \omega=0$, then $\tilde{\omega}=e^{f} \omega$ is a locally conformally symplectic form taming $J$ which satisfies $d_{\tilde{\alpha}} \tilde{\omega}=0$ with $\tilde{\alpha}=\alpha+d f$. It follows that the existence of a locally conformally symplectic form taming $J$ with Lee form $\alpha$ merely depends upon the de Rham class $a=[\alpha]$.

Our first observation is the following lemma. 
6 V. Apostolov and G. Dloussky

Lemma 2.4. Let $\omega$ be a locally conformally symplectic form on $X=(M, J)$ with Lee form $\alpha$. Denote by $F:=\omega^{1,1}$ the $(1,1)$-part of $\omega$. Then,

$$
d_{\alpha} d_{\alpha}^{c} F=0
$$

where $d_{\alpha}^{c}=J d_{\alpha} J^{-1}=i\left(\bar{\partial}_{\alpha}-\partial_{\alpha}\right)$.

Proof. Write

$$
\omega=F+\omega^{2,0}+\omega^{0,2}
$$

where $\omega^{2,0}$ and $\omega^{0,2}$ denote the $(2,0)$ and $(0,2)$-part of $\omega$, respectively. As $d_{\alpha}=\partial_{\alpha}+\bar{\partial}_{\alpha}$, one has

$$
d_{\alpha} \omega=0 \Longleftrightarrow\left\{\begin{array}{l}
\partial_{\alpha} F+\bar{\partial}_{\alpha} \omega^{2,0}=0 \\
\bar{\partial}_{\alpha} F+\partial_{\alpha} \omega^{0,2}=0
\end{array}\right.
$$

hence $2 i \partial_{\alpha} \bar{\partial}_{\alpha} F=d_{\alpha} d_{\alpha}^{c} F=0$.

Lemma 2.5. Let $S=(M, J)$ be a complex surface and $g$ be a Hermitian metric with fundamental 2-form $F(\cdot, \cdot)=g(J \cdot, \cdot)$. Then,

$$
d_{\alpha} d_{\alpha}^{c} F=0 \Longleftrightarrow \delta(\theta-\alpha)+g(\theta-\alpha, \alpha)=0
$$

where $\delta$ is the co-differential with respect to $g$ and $\theta=J \delta F$ is the Lee form of $g$.

Proof. Using $d F=\theta \wedge F$ (which, as $F$ is a self-dual 2-form with respect to $g$, is equivalent to the relation $\theta=J \delta F)$, one gets

$$
d_{\alpha} d_{\alpha}^{c} F=[d(J(\theta-\alpha))+(\theta-\alpha) \wedge J(\theta-\alpha)] \wedge F
$$

As $F$ is self-dual, that is, $* F=F$, where $*$ stands for the Hodge operator with respect to $g, d_{\alpha} d_{\alpha}^{c} F=0$ is equivalent to

$$
\begin{aligned}
0 & =g(d(J(\theta-\alpha))+(\theta-\alpha) \wedge J(\theta-\alpha), F)=\sum_{i=1}^{4}\left(D_{e_{i}}(J(\theta-\alpha))\left(J e_{i}\right)\right)+|\theta-\alpha|_{g}^{2} \\
& =\sum_{i=1}^{4}\left(\left(D_{e_{i}} J\right)(\theta-\alpha)\left(J e_{i}\right)+\left(D_{e_{i}}(\theta-\alpha)\right)\left(e_{i}\right)\right)+|\theta-\alpha|_{g}^{2} \\
& =(\theta-\alpha)\left(J \sum_{i=1}^{4}\left(D_{e_{i}} J\right)\left(e_{i}\right)\right)-\delta(\theta-\alpha)+|\theta-\alpha|_{g}^{2} \\
& =-g(\theta, \theta-\alpha)-\delta(\theta-\alpha)+g(\theta-\alpha, \theta-\alpha)=-\delta(\theta-\alpha)-g(\theta-\alpha, \alpha),
\end{aligned}
$$


where $D$ is the Riemannian connection of $g,\left\{e_{i}, i=1, \ldots, 4\right\}$ is any $J$-adapted orthonormal frame, and for passing from the third line to the fourth we have used the fact that $D_{e_{i}} J$ is skew-symmetric with respect to $g$ and anti-commutes with $J$, whereas for passing from the fourth line to the fifth, we have used the identity $\theta(X)=(\delta F)$ $(-J X)=-\sum_{i=1}^{4} g\left(J\left(D_{e_{i}} J\right)\left(e_{i}\right), X\right)$.

Remark 2.6. In the light of Remark 2.3 , it is easily seen that the condition

$$
\delta(\theta-\alpha)+g(\theta-\alpha, \alpha)=0
$$

is also conformally invariant. More precisely, it is straightforward to check that if $\tilde{g}=e^{f} g$ and $\tilde{\alpha}=\alpha+d f$, then (5) is satisfied for $(g, \alpha)$ if and only if it is satisfied for $(\tilde{g}, \tilde{\alpha})$.

Recall the fundamental result of Gauduchon [17] which affirms that if $X=(M, J)$ is an $m$-dimensional compact complex manifold endowed with a Hermitian metric $g$, then there exists (a unique up to scale) conformal metric $\tilde{g}=e^{f} g$ whose fundamental form $\tilde{F}$ satisfies $d d^{c} \tilde{F}^{m-1}=0$, or equivalently, for which $\tilde{\delta} J \tilde{\delta} \tilde{F}=0$. Such Hermitian metric is referred to as Gauduchon metric. By Lemma 2.5 and Remark 2.6, we then obtain

Proposition 2.7. Let $S=(M, J)$ be a compact complex surface, $a \in H_{d R}^{1}(M)$ be a de Rham class, and $c=[g]$ be a conformal class of Hermitian metrics on $M$, with $g$ a Gauduchon metric in $c$. Then the following conditions are equivalent:

(i) for any $\tilde{g} \in c$, the fundamental 2-form $\tilde{F}$ satisfies $d_{\tilde{\alpha}} d_{\tilde{\alpha}}^{c} \tilde{F}=0$ for some $\tilde{\alpha} \in a$;

(ii) there exists a positive smooth function $\psi$ on $M$ which satisfies the equation

$$
\mathbb{L}_{g, a}(\psi)=\Delta_{g}(\psi)-g\left(\theta^{g}-2 a_{h}^{g}, d \psi\right)+g\left(\theta^{g}-a_{h}^{g}, a_{h}^{g}\right) \psi=0,
$$

where $\Delta_{g}$ is the Riemannian Laplace operator of $g, \theta^{g}$ is the corresponding co-closed Lee form, and $a_{h}^{g}$ is the harmonic representative for $a$ with respect to $g$.

Proof. By Remark 2.6, if, for some metric $\tilde{g} \in C$, the corresponding fundamental form $\tilde{F}$ satisfies $d_{\tilde{\alpha}} d_{\tilde{\alpha}}^{c} \tilde{F}=0$, for any other metric $g=e^{-f} \tilde{g}$ in $c$, the fundamental 2-form $F$ satisfies $d_{\alpha} d_{\alpha}^{c} F=0$ with $\alpha=\tilde{\alpha}-d f$. It follows that the condition (i) is equivalent to the existence of a 1-form $\alpha \in a$ such that the fundamental 2-form of a Gauduchon metric $g \in c$ satisfies 
$d_{\alpha} d_{\alpha}^{c} F=0$. Writing $\alpha=a_{h}^{g}-d \log \psi$ with $\psi>0$, Lemma 2.5 reads

$$
\begin{aligned}
0 & =\delta\left(\theta^{g}-\alpha\right)+g\left(\theta^{g}-\alpha, \alpha\right)=-\delta \alpha+g\left(\theta^{g}-\alpha, \alpha\right) \\
& =-\delta\left(a_{h}^{g}-d \log \psi\right)+g\left(\theta^{g}-a_{h}^{g}+d \log \psi, a_{h}^{g}-d \log \psi\right) \\
& =\Delta_{g}(\log \psi)-g\left(\theta^{g}-2 a_{h}^{g}, d \log \psi\right)+g\left(\theta^{g}-a_{h}^{g}, a_{h}^{g}\right)-g(d \log \psi, d \log \psi) \\
& =\frac{1}{\psi}\left(\Delta_{g} \psi-g\left(\theta^{g}-2 a_{h}^{g}, d \psi\right)+g\left(\theta^{g}-a_{h}^{g}, a_{h}^{g}\right) \psi\right),
\end{aligned}
$$

and the claim follows.

The following elementary observation regarding the linear operator $\mathbb{L}_{g, a}$ will be used throughout.

Lemma 2.8. For any everywhere positive smooth function $\psi$ on $M$

$$
\int_{M} \frac{\mathbb{L}_{g, a}(\psi)}{\psi} v_{g}=\int_{M} g\left(\theta^{g}, a_{h}^{g}\right)-\int_{M}\left(\left\|a_{h}^{g}\right\|_{g}^{2}+\frac{1}{\psi^{2}}\|d \psi\|_{g}^{2}\right) v_{g} .
$$

In particular, if (6) admits a positive solution with respect to some $a \neq 0 \in H_{d R}^{1}(M)$, then

$$
\int_{M} g\left(\theta^{g}, a_{h}^{g}\right) v_{g}=\int_{M} g\left(\theta_{h}^{g}, a_{h}^{g}\right) v_{g}=\int_{M}\left(\left\|a_{h}^{g}\right\|_{g}^{2}+\frac{1}{\psi^{2}}\|d \psi\|_{g}^{2}\right) v_{g}>0 .
$$

Proof. The equality (8) follows by integration by parts and using that $g$ is Gauduchon, that is, $\delta \theta^{g}=0$; the inequality (9) is an immediate consequence.

The general theory for the existence of positive solutions of the elliptic linear second-order PDE $\mathbb{L}_{g, a}(\psi)=0$ is reviewed in Appendix to this paper. We recollect below the following variational characterization.

Proposition 2.9. The PDE (6) has a positive solution $\psi$ if and only if

$$
\lambda_{a}(g):=\sup _{u \in \mathcal{C}^{\infty}(M), u>0}\left\{\min _{M} \frac{\mathbb{L}_{g, a}(u)}{u}\right\}=0 .
$$

Furthermore, $\lambda_{a}(g)$ is a finite number that varies analytically with respect to linear variations $g_{t}=(1-t) g+t \tilde{g}$ of Gauduchon metrics, or linear variations $a_{t}=t a$ of de Rham classes. 
Proof of Theorem 1.2. By Propositions 2.7 and 2.9, it is enough to fix a Gauduchon metric $g \in c$ and show that $\lambda_{a}(g)=0$ for a suitable choice of $a \neq 0$. Let $\theta_{h}^{g}$ denote the harmonic part of the Lee form of $g$. It is well known (see e.g. [3, Proposition 1] or Proposition 3.5) that $\left[\theta_{h}^{g}\right] \neq 0$ under the assumption that the first Betti number is odd. We let $a_{t}=\frac{t}{2}\left[\theta_{h}^{g}\right]$ with $t>0$ and denote by $a_{h}^{t}=\frac{t}{2} \theta_{h}^{g}$ the harmonic representative of $a_{t}$ with respect to $g$. We are going to show that $\lambda_{a_{t}}(g)<0$ when $t \geq 2$ and $\lambda_{a_{t}}(g)>0$ for $t$ close to 0 ; by the second part of Proposition 2.9, this would imply that $\lambda_{a_{t}}(g)=0$ for some $t \in(0,2)$.

Let $u_{t}>0$ be the eigenfunction of $\mathbb{L}_{g, a_{t}}$, corresponding to the principal eigenvalue $\lambda(t):=\lambda_{a_{t}}(g)$, normalized by $\int_{M} u_{t}^{2} v_{g}=1$ (see Theorem A.1). By Lemma 2.8 we have

$$
\begin{aligned}
\lambda(t) \operatorname{vol}_{g}(M) & =\int_{M} \frac{\mathbb{L}_{g, a_{t}}\left(u_{t}\right)}{u_{t}} v_{g}=-\int_{M} \frac{1}{u_{t}^{2}}\left\|d u_{t}\right\|_{g}^{2} v_{g}+\int_{M} g\left(\theta_{h}^{g}-a_{h}^{t}, a_{h}^{t}\right) v_{g} \\
& =-\int_{M} \frac{1}{u_{t}^{2}}\left\|d u_{t}\right\|_{g}^{2} v_{g}+\frac{t(2-t)}{4} \int_{M}\left\|\theta_{h}^{g}\right\|_{g}^{2} v_{g} .
\end{aligned}
$$

Taking $t \geq 2$ in (11) yields $\lambda(t) \leq 0$. We are going to show that $\lambda(t)>0$ for positive $t$ close to 0 . Indeed, as for each $t \lambda(t)$ is a simple eigenvalue (see Theorem A.1), by the KatoRellich theory $[26,41]$ (see Theorem A.3), $\lambda(t)$ and $u_{t}$ vary analytically with respect to $t$. As $\mathbb{L}_{g, 0}$ does not have a zero-order term, by the Hopf maximum principle (see e.g. [4, III, Section 8, 3.71]) $\lambda(0)=0$ and $u_{0}=1 / \operatorname{vol}_{g}(M)$. It thus follows that the function $t \mapsto$ $\int_{M} \frac{\left\|d u_{t}\right\|_{g}^{2}}{u_{t}^{2}} v_{g}$ has a global minimum 0 at $t=0$, so differentiating (11) at $t=0$ we get

$$
\lambda^{\prime}(0) \operatorname{vol}_{g}(M)=\frac{1}{2} \int_{M}\left\|\theta_{h}^{g}\right\|_{g}^{2} v_{g}>0 .
$$

In order to obtain a converse of Lemma 2.4, we first note the following lemma.

Lemma 2.10. Let $\mathcal{L}$ be a flat holomorphic line bundle over a compact complex surface $S$, such that $H^{0}(S, \mathcal{L})=H^{2}(S, \mathcal{L})=\{0\}$. Then, $H_{\bar{\partial}_{\mathcal{L}^{*}}}^{2,1}\left(S, \mathcal{L}^{*}\right)=\{0\}$.

Proof. As $H_{\grave{\partial}_{\mathcal{L}^{*}}}^{2,1}\left(S, \mathcal{L}^{*}\right) \cong H^{1}\left(S, \mathcal{K}_{S} \otimes \mathcal{L}^{*}\right)$, by Serre duality

$$
\operatorname{dim} H_{\bar{\partial}_{\mathcal{L}^{*}}}^{2,1}\left(S, \mathcal{L}^{*}\right)=h^{1}\left(S, \mathcal{K}_{S} \otimes \mathcal{L}^{*}\right)=h^{1}(\mathcal{L})
$$

Using the vanishing of the cohomology groups from the hypothesis of the lemma and the Riemann-Roch formula, one concludes $\operatorname{dim} H_{\bar{\partial}_{\mathcal{L}^{*}}}^{2,1}\left(S, \mathcal{L}^{*}\right)=0$. 
Let $\mathcal{L}_{a}$ be the flat holomorphic line bundle corresponding to $a=[\alpha]$; see Convention 2.1. Its degree with respect to the Gauduchon metric $g$ is defined to be [18]

$$
\operatorname{deg}_{g}\left(\mathcal{L}_{a}\right)=\frac{1}{2 \pi} \int_{M} \rho_{\mathcal{L}_{a}} \wedge F,
$$

where $\frac{1}{2 \pi} \rho_{\mathcal{L}_{a}}$ is a pluriharmonic representative of the first Chern class of $\mathcal{L}_{a}$. Writing $\alpha_{\mid U_{i}}=d f_{i}$ on an open covering $\mathfrak{U}=\left(U_{i}\right)$ of $M, \mathcal{L}_{a}$ is the topologically trivial complex line bundle over $M$ with $s_{0}=\left(U_{i}, e^{f_{i}}\right)$ being a nowhere vanishing smooth section, whereas $h_{\mid U_{i}}=e^{-2 f_{i}}(\cdot, \cdot)$ introduces a Hermitian metric on $\mathcal{L}_{a}$ with $h\left(s_{0}, s_{0}\right)=1$. It follows that $\rho=-\frac{1}{2} d d^{c} \log e^{-2 f_{i}}=-d^{c} \alpha$ is the curvature of the Chern connection of $\left(\mathcal{L}_{a}, h\right)$, so that

$$
\begin{aligned}
\operatorname{deg}_{g}\left(\mathcal{L}_{a}\right) & =-\frac{1}{2 \pi} \int_{M} d^{c} \alpha \wedge F=-\frac{1}{2 \pi} \int_{M} g\left(d^{c} \alpha, F\right) v_{g} \\
& =-\frac{1}{2 \pi} \int_{M} g\left(\theta^{g}, \alpha\right) v_{g}=-\frac{1}{2 \pi} \int_{M} g\left(\theta_{h}^{g}, a_{h}^{g}\right) v_{g},
\end{aligned}
$$

where for the last line we have used that $d \alpha=0, \delta \theta^{g}=0$. It then follows from Proposition 2.7, the inequality (9) of Lemma 2.8 and the properties of the degree (see e.g. [18]).

Lemma 2.11. Let $g$ be a Gauduchon Hermitian metric on a compact complex surface $S$, whose fundamental 2-form $F$ satisfies $d_{\alpha} d_{\alpha}^{c} F=0$ for some closed but not exact 1-form $\alpha$. Then, the flat holomorphic line bundle $\mathcal{L}_{a}$ determined by $a=[\alpha] \in H_{d R}^{1}(M)$ via (1) and Convention 2.1 satisfies

$$
\operatorname{deg}_{g}\left(\mathcal{L}_{a}\right)=-\frac{1}{2} \int_{M}\left(\left\|a_{h}^{g}\right\|_{g}^{2}+\frac{1}{\psi^{2}}\|d \psi\|_{g}^{2}\right) v_{g}<0,
$$

so that $H^{0}\left(S, \mathcal{L}_{a}^{\ell}\right)=\{0\}$ for all $\ell \geq 1$.

We thus obtain the following

Proposition 2.12. Let $g$ be a Gauduchon Hermitian metric on a compact complex surface $S$, whose fundamental 2-form $F$ satisfies $d_{\alpha} d_{\alpha}^{c} F=0$ for some closed but not exact 1 -form $\alpha$. If the holomorphic flat bundle $\mathcal{L}_{a}$ corresponding to the deRham class $a=[\alpha]$ via (1) and Convention 2.1 satisfies $H^{2}\left(S, \mathcal{L}_{a}\right)=\{0\}$, then there exists a locally conformally symplectic 2 -form $\omega$ on $S$ with Lee form $\alpha$ and whose $(1,1)$-part is $F$.

Proof. As $H^{0}\left(S, \mathcal{L}_{a}\right)=\{0\}$ by Lemma 2.11, the hypothesis $H^{2}\left(S, \mathcal{L}_{a}\right)=\{0\}$, Lemma 2.10 and the isomorphism (4) imply $H_{\bar{\partial}_{\mathcal{L}_{a}^{*}}^{*}}^{2,1}\left(S, \mathcal{L}_{a}^{*}\right) \cong H_{\bar{\partial}_{\alpha}}^{2,1}(S)=\{0\}$. As $\bar{\partial}_{\alpha} \partial_{\alpha} F=\frac{i}{2} d_{\alpha} d_{\alpha}^{c} F=0$, it follows that

$$
\partial_{\alpha} F+\bar{\partial}_{\alpha} \beta=0
$$


for a $(2,0)$-form $\beta$. Letting

$$
\omega=F+\beta+\bar{\beta}
$$

one has

$$
d_{\alpha} \omega=\left(\partial_{\alpha}+\bar{\partial}_{\alpha}\right)(F+\beta+\bar{\beta})=\partial_{\alpha} F+\bar{\partial}_{\alpha} \beta+\bar{\partial}_{\alpha} F+\partial_{\alpha} \bar{\beta}=0
$$

which completes the proof.

Proof of Theorem 1.1. Let $S=(M, J)$ be a compact complex surface with odd first Betti number $b_{1}(M)$. If the Kodaira dimension of $S$ is non-negative, it follows from [6, 47] that $S$ admits a locally conformally Kähler metric. We therefore suppose that the Kodaira dimension of $S$ is negative, that is, $S$ belongs to class VII of the Kodaira list; see for example, [5]. Denote by $S_{0}$ the minimal model of $S$.

We first suppose that the second Betti number of $S_{0}$ is greater than or equal to 1 , that is, $S_{0}$ is in the Kodaira class $\mathrm{VII}_{0}^{+}$(for which a complete classification is still to come). In this case, Theorem 1.1 follows from Theorem 1.2, Proposition 2.12, and the following vanishing result.

Lemma 2.13. Let $S$ be a compact complex surface with $b_{1}(S)=1$ and negative Kodaira dimension. Suppose that the second Betti number of its minimal model $S_{0}$ is $>0$. Then, for any topologically trivial line bundle $\mathcal{L} \in \operatorname{Pic}_{0}(S)$, we have

$$
H^{2}(S, \mathcal{L})=0
$$

Proof. By Serre duality, we have to show that, for any $\mathcal{L} \in \operatorname{Pic}_{0}(S), H^{0}\left(S, \mathcal{K}_{S} \otimes \mathcal{L}\right)=0$. Suppose that there exists a non-trivial section $\sigma \in H^{0}\left(S, \mathcal{K}_{S} \otimes \mathcal{L}\right)$. The line bundle $\mathcal{K}_{S} \otimes \mathcal{L}$ is not trivial as otherwise $\mathcal{K}_{S}^{-1}$ must be flat, and therefore $0=c_{1}^{2}(S)=-b_{2}(S)=-c_{2}(S)$ (see [5]), which is a contradiction. It follows that $\sigma$ must vanish along an effective divisor $D$ with $[D]=\mathcal{K}_{S} \otimes \mathcal{L}$. Therefore, in $H^{2}(S, \mathbb{Z})$,

$$
0=\left(\mathcal{K}_{S}+\mathcal{L}-D\right) \cdot \mathcal{K}_{S}=\mathcal{K}_{S}^{2}-\mathcal{K}_{S} \cdot D
$$

We show the assertion by induction on the number $p$ of blowing ups.

If $p=0$, the surface is minimal and by [34, p. 399], an irreducible curve $C$ of $D$ is either

- a rational curve such that $C^{2} \leq-2$, and therefore, by the adjunction formula, $0=\pi(C)=1+\frac{\mathcal{K}_{S} \cdot C+C^{2}}{2}$ and $K_{S} \cdot C \geq 0$, or 
- an elliptic or a rational curve $C$ with a double point. By the same formula, $\mathcal{K}_{S} \cdot C+C^{2}=0$, hence $K_{S} \cdot C \geq 0$,

We deduce that, for any effective divisor $D, \mathcal{K}_{S} \cdot D \geq 0$. Moreover, as $b_{2}(S)=-C_{1}(S)^{2}=$ $-\mathcal{K}_{S}^{2}>0$, we have a contradiction in (13).

If $p \geq 1$, there is an exceptional curve of the first kind $E$ and let $B_{X}: S \rightarrow \check{S}$ be the blowing down of $E$ to a point $x \in \check{S}$. Let $\check{U}$ be a ball centred at $x$ and $U=B_{X}{ }^{-1}(\check{U})$ be a simply connected neighborhood of $E$. The line bundle $\mathcal{L}$ is holomorphically trivial on $U$ and, therefore, the coherent sheaf $\breve{\mathcal{L}}=\left(B_{X}\right)_{*} \mathcal{L}$ is in fact a topologically trivial line bundle on $\check{S}$. As $\left(B_{X}\right)_{*} \mathcal{K}_{S}=\mathcal{K}_{\check{S}}$, any non-trivial section $\sigma \in H^{0}\left(S, \mathcal{K}_{S} \otimes \mathcal{L}\right)$ gives, via the biholomorphism $B_{X}: S \backslash E \cong \check{S} \backslash\{x\}$ a non-trivial section $\check{\sigma} \in H^{0}\left(\check{S} \backslash\{x\}, \check{\mathcal{K}}_{\check{S}} \otimes \check{\mathcal{L}}\right)$. By Hartogs' theorem, this section extends to $\check{S}$. As $b_{2}(\check{S}) \geq b_{2}\left(S_{0}\right)>0$, by the induction hypothesis $\check{\sigma}=0$, which is a contradiction.

Let us now consider the case when $b_{2}\left(S_{0}\right)=0$. According to $[8,33,44], S_{0}$ is either an Inoue surface (see [24]) or a Hopf surface (see [25]). The arguments in [47, 48] can be used without any change to show that the blow-up $S$ of $S_{0}$ admits a locally conformally symplectic form taming $J$ if $S_{0}$ does. We thus consider the case $S=S_{0}$.

The Inoue surfaces with second Betti number equal to zero are classified by Inoue [24] who shows that they do not admit curves. Thus, if $S$ is such a surface, the condition $H^{2}(S, \mathcal{L}) \cong H^{0}\left(S, \mathcal{K}_{S} \otimes \mathcal{L}^{*}\right)=\{0\}$ is equivalent to $\mathcal{L} \neq \mathcal{K}_{S}$, where $\mathcal{K}_{S}$ denotes the canonical bundle of $S$. Let $g$ be a Gauduchon metric whose fundamental 2-form $F$ satisfies $d_{\alpha} d_{\alpha}^{c} F=0$ for a 1 -form $\alpha$ with $a=[\alpha] \neq 0$, given by Theorem 1.2. The corresponding line bundle $\mathcal{L}=\mathcal{L}_{a}$ has negative degree with respect to $g$ by (12), whereas it is shown in [45, Remark 4.2] that the degree of $\mathcal{K}_{S}$ is positive with respect to any Gauduchon metric. Thus, $\mathcal{L} \neq \mathcal{K}_{S}$, showing that $H^{2}(S, \mathcal{L})=\{0\}$, and therefore $S$ admits a locally conformally symplectic 2-form taming $J$ with Lee form $\alpha$ by Proposition 2.12.

If $S$ is a Hopf surface, Lemma 2.13 generally fails, so we cannot directly use Theorem 1.2 in this case. However, according to $[6,19], S$ admits an lcK metric.

\section{Cohomological Invariants of a non-Kähler Complex Manifold}

An important and well-studied invariant associated to a compact complex manifold $X=(M, J)$ which admits Kähler metrics is its Kähler cone, $\mathcal{K}(X)$, defined to be the subset of classes $\Omega \in H_{d R}^{1,1}(X, \mathbb{R})$ for which there exists a Kähler metric on $X$ whose fundamental 2 -form belongs to $\Omega$. A characterization of $\mathcal{K}(X)$ in terms of the intersection form of the cohomology ring of $X$, the Hodge structure, and homology of analytic cycles has been obtained by Buchdahl $[11,12]$ and Lamari $[30,31]$ when $X$ is a compact complex surface, 
and Demailly-Paun [13] in general. These results imply in particular that the classes in $H_{d R}^{1,1}(X)$ which contain symplectic forms taming $J$ coincide with $\mathcal{K}(X)$.

In order to introduce similarly designed cohomological invariants in the nonKähler lcK case, one can consider the sets (see [9, 38]) as follows.

Definition 3.1. Let $X=(M, J)$ be a compact complex manifold. We introduce the following subsets of $H_{d R}^{1}(M)$ :

- The subset of classes of Lee forms of lcK metrics:

$$
\mathcal{C}(X)=\left\{[\alpha] \mid \exists F \in \Omega^{1,1}(X), F>0, d_{\alpha} F=0\right\} .
$$

- The subset of classes of Lee forms of locally conformally symplectic forms taming $J$ :

$$
\mathcal{T}(X)=\left\{[\alpha] \mid \exists \omega \in \Omega^{2}(X), \omega^{1,1}>0, d_{\alpha} \omega=0\right\} .
$$

- The subset of classes of the harmonic parts of the Lee forms of Gauduchon metrics

$$
\mathcal{G}(X)=\left\{\left[\theta_{h}^{g}\right] \mid \exists F \in \Omega^{1,1}(X), F>0, d d^{c} F^{m-1}=0, d_{\theta} F^{m-1}=0\right\},
$$

where $\theta_{h}^{g}$ denotes the harmonic part of $\theta$ with respect to the Riemannian met$\operatorname{ric} g(\cdot, \cdot)=F(\cdot, J \cdot)$.

Remark 3.2. It follows from the definition that

$$
\mathcal{C}(X) \subseteq \mathcal{T}(X), \quad \mathcal{C}(X) \subseteq \mathcal{G}(X)
$$

$\mathcal{G}(X)$ is connected while $\mathcal{T}(X)$ is invariant under small deformations of $X$. Using similar techniques as in [20], one can show that $a \in \mathcal{T}(X)$ is an interior point, provided that $H_{d_{L^{*}}}^{3}\left(M, L^{*}\right)=\{0\}$, where $L=L_{a}$ is the real flat line bundle determined by $a$.

We now consider the blow-up $\hat{X}$ of $X$ at a point $x$ and denote by $B_{X} \hat{X} \rightarrow X$ the blow-down map which is a biholomorphism between $\hat{X} \backslash E \rightarrow X \backslash\{X\}$, where $E \cong \mathbb{C} P^{m-1}$ is the exceptional divisor. The following lemma is well-known.

Lemma 3.3. Let $B_{X}: \hat{X} \rightarrow X$ be the blow-down map which contracts a divisor $E \cong$ $\mathbb{C} P^{m-1} \subset \hat{X}$ with normal bundle $N_{E} \cong \mathcal{O}(-1)$ to a point $x \in X$. For any $a \in H_{d R}^{1}(X)$ with generator $\alpha \in a$, denote by $\hat{\alpha}=B_{X}^{*}(\alpha)$. Then $B_{X}^{*}: H_{\alpha}^{k}(X) \rightarrow H_{\hat{\alpha}}^{k}(\hat{X})$ is surjective for any positive $k \neq 2(m-1)$, and is injective for any positive $k \neq 2 m-1$. 
Proof. As $H_{\alpha}^{k}(X)$ does not depend on the choice of $\alpha \in a$ (see the discussion in Section 2), we can choose $\alpha$ such that it identically vanishes on a open ball $U$ centred at $x$. It follows that $\hat{\alpha}=B_{X}^{*}(\alpha)$ vanishes on $\hat{U}=B_{X}^{-1}(U)$.

We will first prove that $B_{X}^{*}: H_{\alpha}^{k}(X) \rightarrow H_{\hat{\alpha}}^{k}(\hat{X})$ is surjective. With our choice for $\alpha$, any $d_{\hat{\alpha}}$-closed $k$-form $\hat{\varphi}$ on $\hat{X}$ is closed over $\hat{U}$. As $H_{d R}^{k}(\hat{U}) \cong H_{d R}^{k}\left(\mathbb{C} P^{m-1}\right)=\{0\}$ for $k \neq 2$ $(m-1)$, we can write $\hat{\varphi}_{\mid \hat{U}}=d\left(\hat{\xi}_{\mid \hat{U}}\right)$. Multiplying $\hat{\xi}_{\mid \hat{U}}$ by the pull-back via $B_{X}$ of a bump function centred at $x$ and support in $U$, we can assume that $\hat{\xi}$ is globally defined on $\hat{X}$ and $\hat{\phi}=\hat{\varphi}-d_{\hat{\alpha}} \hat{\xi}$ is another form representing $[\hat{\varphi}] \in H_{\hat{\alpha}}^{k}(\hat{X})$, which vanishes identically on a tubular neighborhood of $E$. Then, the diffeomorphism $\left(B_{X}^{-1}\right): \hat{X} \backslash E \rightarrow X \backslash\{x\}$ allows one to define a smooth $k$-form $\phi=\left(B_{X}^{-1}\right)^{*}(\hat{\phi})$ on $X$ with $d_{\alpha} \phi=0$ and $B_{X}^{*}(\phi)=\hat{\phi}$.

We now prove that $B_{X}^{*}: H_{\alpha}^{k}(X) \rightarrow H_{\hat{\alpha}}^{k}(\hat{X})$ is injective. Suppose that $\varphi$ is a $d_{\alpha}$-closed $k$-form on $X$, such that $\hat{\varphi}=B_{X}^{*}(\varphi)=d_{\hat{\alpha}} \hat{\xi}$. As $H_{d R}^{k}(U)=\{0\}$, we can modify $\varphi$ with a $d_{\alpha}$-exact form (as we did above with $\hat{\varphi}$ ) and assume without loss that $\varphi_{\mid U} \equiv 0$. It follows that the $(k-1)$-form $\hat{\xi}$ satisfies $d \hat{\xi}_{\mid \hat{U}} \equiv 0$. If $k=1, \hat{\xi}$ is a smooth function on $\hat{X}$, which is constant on $\hat{U}$ and, therefore, is the pull-back to $\hat{X}$ of a smooth function $\xi$ on $X$ (which is constant on $U)$. It follows that $\varphi=d_{\alpha} \xi$. If $k>1, H_{d R}^{k-1}(\hat{U}) \cong H^{k-1}\left(\mathbb{C} P^{m-1}\right)=\{0\}$, so we conclude that $\hat{\xi}_{\mid \hat{U}}$ is exact, that is, $\hat{\xi}_{\hat{U}}=d \hat{\eta}_{\hat{U}}$. Multiplying $\hat{\eta}$ by a bump-function, we obtain that $\hat{\xi}-d_{\hat{\alpha}} \hat{\eta}$ is identically zero in a neighborhood of $E$, so that it descends to $X$ to define a $(k-1)$-form $\xi$ with $d_{\alpha} \xi=\varphi$.

We recall the following result established in [47,48]. The argument in [48] applies without change to the case of locally conformally symplectic structures taming $J$.

Proposition 3.4. Let $\hat{X}$ be the blow-up of $X$ at a point $x$. Then

(a) if $a \in \mathcal{T}(X)$, then $\hat{a}=B_{X}^{*}(a) \in \mathcal{T}(\hat{X})$;

(b) $\quad a \in \mathcal{C}(X)$ if and only if $\hat{a}=B_{X}^{*}(a) \in \mathcal{C}(\hat{X})$.

When $X=S$ is a compact complex surface, the following result is well known.

Proposition 3.5. On a compact complex surface $S=(M, J)$ the following conditions are equivalent:

(i) $\quad 0 \in \mathcal{C}(S)$, that is, $S$ is Kähler;

(ii) $0 \in \mathcal{T}(S)$;

(iii) $0 \in \mathcal{G}(S)$;

(iv) $\mathcal{C}(S)=\mathcal{T}(S)=\mathcal{G}(S)=\{0\}$. 
Proof. “(i) $\Rightarrow$ (ii)" and "(iv) $\Rightarrow$ (i)" are obvious. In order to prove "(ii) $\Rightarrow$ (iii)", let $\omega$ be a symplectic 2-form on $M$ which tames $J$. Letting $F=\omega^{1,1}$ be the positive-definite $(1,1)$-part, it defines a Hermitian metric $g(\cdot, \cdot)=F(\cdot, J \cdot)$ which is Gauduchon (see e.g. Lemma 2.4 with $\alpha=0$ ). Furthermore, $\omega$ is a closed self-dual 2-form with respect to $g$, and is therefore co-closed. Writing $\omega=F+\Psi$, where $\Psi$ is of type $(2,0)+(0,2)$, we get

$$
0=\delta \omega=\delta F+\delta \Psi=-J \theta^{g}-J(\delta \mathcal{J} \Psi)
$$

where $\mathcal{J}$ stands for the natural action of the almost-complex structure $J$ on the bundle of real 2 -forms of type $(2,0)+(0,2)$ by $\mathcal{J} \Psi(X, Y):=-\Psi(J X, Y)$. This shows that the Lee form $\theta$ of $g$ is co-exact and, therefore, $\theta_{h}^{g}=0$.

It remains to establish "(iii) $\Rightarrow$ (iv)". To this end, by [3, Proposition 1], $b_{1}(M)$ is even and $\mathcal{G}(S)=\{0\}$. It follows that $\mathcal{C}(S)=\{0\}$ as $\mathcal{C}(S) \subseteq \mathcal{G}(S)$ and $\mathcal{C}(S) \neq \emptyset$ by the characterization of Kähler surfaces [11, 30, 42, 46].

In order to prove $\mathcal{T}(S)=\{0\}$, we use Lemma 2.4, Proposition 2.7, and Lemma 2.8: as $\theta_{h}^{g}=0$ in our case, one gets $0 \leq-\int_{M}\left\|a_{h}^{g}\right\|_{g}^{2} v_{g}$, showing $a_{h}^{g}=0$.

Recall the following definition from [36].

Definition 3.6. An $l c K$ metric with potential $g$ on $X=(M, J)$ is an lcK metric such that the pull-back $\tilde{F}$ of its fundamental 2-form $F$ to the universal covering space $\tilde{X}$ of $X$ is of the form $\tilde{F}=\frac{d d^{c} \tilde{f}}{\tilde{f}}$, where $\tilde{f}>0$ is a positive plurisubharmonic function on $\tilde{X}$ which satisfies $\gamma^{*} \tilde{f}=e^{c_{\gamma}} \tilde{f}\left(c_{\gamma} \in \mathbb{R}\right)$ for any deck-transform $\gamma \in \pi_{1}(X)$.

Examples of lcK metrics with potential include the Vaisman lcK metrics (i.e. lcK metrics for which the Lee form $\theta$ is parallel) or more generally, pluricanonical lcK metrics, introduced and studied by Kokarev [29], for which the covariant derivative $D \theta$ of the Lee form is of type $(2,0)+(0,2)$ with respect to $J$; see [36, Claim 3.3; 39]. (In [36], the authors claim that an lcK metric admits a potential if and only if it is pluricanonical, but a proof is given only in one direction; see [39] for the precise link between the two notions.) The following observation is taken from [38].

Lemma 3.7. Let $X=(M, J)$ be a compact complex manifold endowed with an lcK metric with potential, $g$, and Lee form $\theta$. Then, for any $t \geq 1, t \theta$ is the Lee form of an lcK metric with potential on $X$. If, furthermore, $g$ is a pluricanonical lcK metric, then $t \theta$ is the Lee form of a pluricanonical lcK metric for any $t>0$. 
Proof. Writing $\tilde{F}=\frac{d d^{c} \tilde{f}}{\tilde{f}}$ on $\tilde{X}$, the pull-back of the Lee form is $\tilde{\theta}=-\frac{d \tilde{f}}{\tilde{f}}$. For any $t \geq 1$ put

$$
\tilde{F}_{t}:=\frac{d d^{c} \tilde{f}^{t}}{\tilde{f}^{t}}=t \frac{d d^{c} \tilde{f}}{\tilde{f}}+t(t-1) \frac{d \tilde{f} \wedge d^{c} \tilde{f}}{\tilde{f}^{2}} .
$$

For $t \geq 1, \tilde{F}_{t}$ defines a positive-definite $(1,1)$-form satisfying $d \tilde{F}_{t}=\tilde{\theta}_{t} \wedge \tilde{F}_{t}$ with $\tilde{\theta}_{t}=-t \frac{d \tilde{f}}{\tilde{f}}=$ $t \tilde{\theta}$. As $\tilde{F}_{t}$ is invariant under any deck transformation, it defines an lcK metric with potential, $g_{t}$, on $X$, whose Lee form is $\theta_{t}=t \theta$.

It is not hard to see that the pluricanonical condition $\left(D_{X}^{g} \theta\right)(Y)=-\left(D_{J X}^{g} \theta\right)(J Y)$ is equivalent to

$$
d J \theta=-|\theta|_{g}^{2} F+\theta \wedge J \theta .
$$

This is essentially the formula appearing in [36, p. 724], by noting that there is a sign error in [36] in deriving the formula for $d(I \theta)$ from the previous one, and an omission of a factor $|\theta|_{g}^{2}$ before $g$ in the formula expressing $\nabla \theta-D \theta$; the precise statement appears in [39]. For convenience of the reader we supply here a brief argument for (14): it is well known (see e.g. [27, II, Proposition 4.2]) that when $J$ is integrable

$$
\left(D_{X} F\right)(Y, Z)=-\frac{1}{2}(d F(X, J Y, J Z)-d F(X, Y, Z))
$$

The lcK condition implies $d F=\theta \wedge F$, which allows one to rewrite the above equality as

$$
D_{X} F=\frac{1}{2}\left(X^{\triangleright} \wedge J \theta+J X^{\triangleright} \wedge \theta\right)
$$

where $X^{\downarrow}$ denotes the $g$-dual 1 -form to $X$. Thus, using (15),

$$
\begin{aligned}
\left(D_{X} J \theta\right)(Y) & =-\theta\left(\left(D_{X} J\right)(Y)\right)-\left(D_{X} \theta\right)(J Y) \\
& =\frac{1}{2}\left(\theta(X) J \theta(Y)-J \theta(X) \theta(Y)-|\theta|_{g}^{2} F(X, Y)\right)-\left(D_{X} \theta\right)(J Y) .
\end{aligned}
$$

It then follows

$$
d J \theta=-|\theta|_{g}^{2} F+\theta \wedge J \theta+(J D \theta)^{\text {anti }}
$$

where $(J D \theta)^{\text {anti }}$ denotes the anti-symmetrization of the $(2,0)$-tensor $(J D \theta)(X, Y):=$ $-D \theta(X, J Y)$; as $d \theta=0$ (i.e. $D \theta$ is symmetric), $J D \theta^{\text {anti }}(X, Y)=-(D \theta)^{1,1}(X, J Y)$ where $D \theta^{1,1}(X, Y):=\left(D_{X} \theta\right)(Y)+\left(D_{J X} \theta\right)(J Y)$. Thus, for an lcK metric $g$, equation (14) is equivalent to $D \theta^{1,1}=0$, that is, to $g$ being pluricanonical. 
Differentiating (14) one more time yields

$$
d|\theta|_{g}^{2} \wedge F=0
$$

showing that $|\theta|_{g}^{2}$ is a constant. Thus

$$
F_{t}:=F+\frac{t}{|\theta|_{g}^{2}} \theta \wedge J \theta, \quad t>-1
$$

defines a family of positive-definite $(1,1)$-forms with $d F_{t}=(1+t) \theta \wedge F_{t}$. Clearly, $F_{t}$ give rise to a family of lcK metrics which verify the pluricanonical condition (14).

Remark 3.8. If $g$ is a pluricanonical lcK (non-Kähler) metric on $X=(M, J)$ with Lee form $\theta$, writing the pull-back metric as $\tilde{g}=e^{\tilde{\varphi}} \tilde{g}_{K}$ where $\tilde{g}_{K}$ is a Kähler metric on the universal cover $\tilde{X}$ conformal to $\tilde{g}$ (or, equivalently, writing the pull-back $\tilde{\theta}$ of the Lee form as $\tilde{\theta}=d \tilde{\varphi}$ ) gives rise to a potential function $\tilde{f}:=e^{-\tilde{\varphi}}$ for the fundamental 2-form $\tilde{F}$ of $\tilde{g}$, that is, $\tilde{F}=\frac{d d^{c} \tilde{f}}{\tilde{f}}$ by (14).

\section{Compact Complex Surfaces with $b_{1}(S)=1$}

We start with the following easy consequence of Proposition 3.5.

Lemma 4.1. Let $S=(M, J)$ be a compact complex surface with $b_{1}(M)=1$. For any $a \in H_{d R}^{1}(M)$, denote by $\mathcal{L}_{a}$ the corresponding flat holomorphic line bundle defined via (1) and Convention 2.1. Then, the sign of $\operatorname{deg}_{g}\left(\mathcal{L}_{a}\right)$ does not depend on the choice of a Gauduchon metric on $S$ and is zero if and only if $a=0$.

Proof. Let $g$ be a Gauduchon metric on $S$. By (12), $\operatorname{deg}_{g}\left(\mathcal{L}_{a}\right)=0$ for any Gauduchon metric if $a=0$. On the other hand, if $a \neq 0, \theta_{h}^{g}=\mu_{a}(g) a_{h}^{g}$ for a constant $\mu_{a}(g)$, so that by (12) again, the sign of $\operatorname{deg}_{g}\left(\mathcal{L}_{a}\right)$ is equal to the sign of $-\mu_{a}(g)$. We know by Proposition 3.5 that $\mu_{a}(g) \neq 0$. As the space of Gauduchon metrics is convex (and therefore connected), it follows that the sign of $\mu_{a}(g)$ is non-zero and is independent of $g$.

The above lemma suggests the following definition.

Definition 4.2. Let $S=(M, J)$ be a compact complex surface with $b_{1}(M)=1$. For $a$, $b \in H_{d R}^{1}(M)$ we will say that $a>b$ if

$$
\operatorname{deg}_{g}\left(\mathcal{L}_{a} \otimes \mathcal{L}_{b}^{*}\right)=\operatorname{deg}_{g}\left(\mathcal{L}_{(a-b)}\right)=\operatorname{deg}_{g}\left(\mathcal{L}_{a}\right)-\operatorname{deg}_{g}\left(\mathcal{L}_{b}\right)>0,
$$

for some (and hence any) Gauduchon metric $g$. 
We will thus identify the ordered set $H_{d R}^{1}(S) \cong(\mathbb{R},>)$.

Proposition 4.3. Let $S=(M, J)$ be a compact complex surface with $b_{1}(M)=1$. Then $\mathcal{G}(S) \subseteq(-\infty, 0)$ and $\mathcal{T}(S) \subseteq(-\infty, 0)$. Furthermore, for each $a \in \mathcal{T}(S)$, there exists a class $b \leq a$ which belongs to $\mathcal{G}(S)$.

Proof. Let $g$ be Gauduchon metric on $S$ and $\theta_{h}^{g}$ be the harmonic part of the corresponding Lee form $\theta^{g}$. Applying (12) for $a=\left[\theta_{h}^{g}\right]$ (and using Proposition 3.5) yields $\operatorname{deg}_{g}\left(\mathcal{L}_{a}\right)=$ $-\frac{1}{2 \pi} \int_{M}\left\|\theta_{h}^{g}\right\|_{g}^{2} v_{g}<0$, thus showing the first inclusion. The second inclusion follows from Proposition 3.5, and Lemmas 2.4 and 2.11. The inequality $\int_{M} g\left(\theta_{h}^{g}-a_{h}^{g}, a_{h}^{g}\right) v_{g}=$ $\int_{M}\left(\frac{1}{\psi}\|d \psi\|_{g}^{2}\right) v_{g} \geq 0$ (see Lemma 2.8) and the fact that $a$ and $b=\left[\theta_{h}^{g}\right]$ are both negative (so that $\theta_{h}^{g}=\mu a_{h}^{g}$ with $\mu>0$ ) show $\operatorname{deg}_{g}\left(\mathcal{L}_{a-b}\right) \geq 0$, that is, the de Rham class $b \leq a$.

Proposition 4.4. Suppose $S=(M, J)$ is a compact complex surface with $b_{1}(M)=1$. If $b \in \mathcal{C}(S), c \in \mathcal{G}(S)$ with $b \leq c$, then any $a \in[b, c] \subset H_{d R}^{1}(S)$, such that the corresponding flat line bundle $\mathcal{L}_{a}$ satisfies $H^{2}\left(S, \mathcal{L}_{a}\right)=\{0\}$, belongs to $\mathcal{T}(S)$.

Proof. Let $g$ be an lcK metric on $S$ whose closed Lee form $\theta^{g}$ belongs to $b$, and suppose (without loss) that $g$ is a Gauduchon metric: thus, the Lee form of $g$ is harmonic, that is, $\theta^{g}=b_{h}^{g}$. For any $a \in H_{d R}^{1}(M)$, let $a_{h}^{g}$ denote the harmonic representative of $a$ with respect to $g$ : thus, writing $a_{h}^{g}=\mu b_{h}^{g}$ for a constant $\mu$, we have $a \geq b$ iff

$$
\operatorname{deg}_{g}\left(\mathcal{L}_{b-a}\right)=-\frac{1}{2 \pi} \int_{M} g\left(b_{h}^{g}, b_{h}^{g}-a_{h}^{g}\right) v_{g}=\frac{(\mu-1)}{2 \pi} \int_{M}\left\|b_{h}^{g}\right\|_{g}^{2} v_{g} \leq 0,
$$

that is, iff $\mu \in[0,1]$. Thus, for any $a \in[b, c]$,

$$
\mathbb{L}_{g, a}(\psi)=\Delta_{g} \psi+(2 \mu-1) g\left(b_{h}^{g}, d \psi\right)+(1-\mu) \mu\left\|b_{h}^{g}\right\|_{g}^{2} \psi
$$

We claim that $\lambda_{a}(g) \geq 0$. Indeed,

$$
\lambda_{a}(g) \geq \min _{M} \mathbb{L}_{g, a}(1)=(1-\mu) \mu \min _{M}\left\|b_{h}^{g}\right\|_{g}^{2} \geq 0 .
$$

Similarly, let $\tilde{g}$ be a Gauduchon metric on $S$ for which the de Rham class $c=\left[\tilde{\theta}_{h}^{\tilde{g}}\right]$ of the harmonic part of its the Lee form satisfies $c \geq b$. For any $a \leq c$, we have $\tilde{\theta}_{h}^{\tilde{g}}=v a_{h}^{\tilde{g}}$ for a real constant $v \in[0,1]$. It then follows that

$$
\int_{M} \tilde{g}\left(\tilde{\theta}^{\tilde{g}}-a_{h}^{\tilde{g}}, a_{h}^{\tilde{g}}\right) v_{\tilde{g}}=(v-1) \int_{M}\left\|a_{h}^{\tilde{g}}\right\|_{\tilde{g}}^{2} v_{\tilde{g}} \leq 0 .
$$

As we saw in the proof of Theorem 1.2 , this implies $\lambda_{a}(\tilde{g}) \leq 0$. 
Considering the linear path $g_{t}=(1-t) g+t \tilde{g}, t \in[0,1]$ of Gauduchon metrics and using the continuity of $\lambda_{a}\left(g_{t}\right)$ (see Proposition 2.9), one concludes that there exists a Gauduchon metric $g^{\prime}$ on $S$ with $\lambda_{a}\left(g^{\prime}\right)=0$. Our claim then follows from Proposition 2.12.

\section{Examples}

In this section, we illustrate the previous discussion on the known compact complex surfaces $S$ in the Kodaira class VII [5]. Note that in this case, $b_{1}(S)=1$.

\subsection{Hopf surfaces}

These are, by definition, compact complex surfaces with universal covering space $\mathbb{C}^{2} \backslash$ $\{(0,0)\}$. It is shown by Kodaira [28] that the fundamental group $\Gamma$ of such a surface is a finite extension of the infinite cyclic group $\mathbb{Z}$. The list of concrete realizations of $\Gamma$ as a group of automorphisms of $\mathbb{C}^{2}$ can be found in [25], and we summarize this classification in the following rough form: $\Gamma=H \ltimes\left\langle\gamma_{0}\right\rangle$, where $\left\langle\gamma_{0}\right\rangle$ denotes the infinite cyclic group generated by the contraction $\gamma_{0}$ of $\mathbb{C}^{2}$

$$
\gamma_{0}\left(z_{1}, z_{2}\right)=\left(\alpha z_{1}+\lambda z_{2}^{m}, \beta z_{2}\right),
$$

where the complex numbers $\alpha, \beta, \lambda$ satisfy $0<|\alpha| \leq|\beta|<1, \lambda\left(\alpha-\beta^{m}\right)=0$ for an integer $m \in \mathbb{N}^{*}$. Furthermore, it follows by the classification in [25] that when $\lambda \neq 0, H \subset$ $\mathrm{U}(1) \times \mathrm{U}(1)$ is abelian and commutes with $\gamma_{0}$. We denote by $S_{\alpha, \beta, \lambda}=\mathbb{C}^{2}\{(0,0)\} /\left\langle\gamma_{0}\right\rangle$ the corresponding primary Hopf surface and by $S_{\alpha, \beta, \lambda ; H}$ the further quotient by $H$, called secondary Hopf surface.

Hopf surfaces with $\lambda=0$ are called diagonal (or, confusingly, of class 1). Belgun has shown [6, Theorem 1] that any such surface admits a Vaisman lcK metric. By Lemma 3.7 and Proposition 4.3, in this case we have $\mathcal{T}(S)=\mathcal{C}(S)=(-\infty, 0)$.

Hopf surfaces with $\lambda \neq 0$ are called resonant (also called, even more confusingly, of class 0). Let $S_{\beta^{m}, \beta, \lambda ; H}$ be a resonant Hopf surface. The analytic family $S_{\lambda}:=S_{\beta^{m}, \beta, \lambda ; H}$, $\lambda \in \mathbb{C}$ has as central fibre the diagonal Hopf surface $S_{0}=S_{\beta^{m}, \beta, 0 ; H}$, while for $\lambda \neq 0$ the surfaces $S_{\lambda}$ are isomorphic (see [19]). As $S_{0}$ admits a taming conformally symplectic form with Lee form in any $a \in(-\infty, 0)$ so does $S_{\lambda}$, just by continuity using that the taming condition is open. It follows that $\mathcal{T}\left(S_{\lambda}\right)=(-\infty, 0)$.

Similarly, as $S_{0}$ admits a Vaisman metric by [6, 19], it has a Vaisman lcK metric $g_{a}$ with fundamental 2-form $F_{a}$ and Lee form in $a$ for any $a \in(-\infty, 0)$, by Lemma 3.7. 
(Recall that any Vaisman lcK metric is pluricanonical.) As the corresponding $H$ commutes with $\gamma_{0}$ in this case, the potential $\tilde{f}$ for $F_{a}$ can be chosen to be $H$-invariant (by averaging over $H$ ). By the argument in [19], the $\tilde{f}$ can be deformed to define an $H$ invariant potential for an lcK metric on $S_{\lambda}$, for $\lambda$ small enough, with the same constant $c_{\gamma_{0}}$ (see Definition 3.6). Using the isomorphism $\mathbb{C}^{*}=H^{1}\left(S, \mathbb{C}^{*}\right) \cong \operatorname{Pic}_{0}(S)$ established for class VII surfaces in [28, I, p. 756], this shows that the induced lcK metrics on $S_{\lambda}$ will have Lee forms in the same de Rham class $a$. We thus see that $\mathcal{C}\left(S_{\lambda}\right)=(-\infty, 0)$ too.

Using Proposition 3.4 (and Lemma 3.3 with $a=0$ and $k=1$ ), we conclude the following proposition.

Proposition 5.1. For any compact complex surface $S$ whose minimal model is a Hopf surface, $\mathcal{T}(S)=\mathcal{C}(S)=(-\infty, 0)$.

\subsection{Inoue surfaces with $b_{2}=0$}

The Inoue surfaces $S_{0}$ with second Betti number equal to zero are classified by Inoue [24] into three types, $S_{M}, S_{N ; p, q, r}^{-}$and $S_{N ; p, q, r ; u^{\prime}}^{+}$where the parameters $M, N$ are matrices with integer coefficients, $p, q, r$ are integers, and $u$ is a complex number. Any such surface is the quotient of $\mathbb{C} \times \mathbb{H}$ (where $\mathbb{H}$ denotes the upper half-plane in $\mathbb{C}$ ) under a discrete subgroup $\Gamma$ of the group $A(2, \mathbb{C})$ of affine transformations of $\mathbb{C}^{2}$, leaving $\mathbb{C} \times \mathbb{H}$ invariant. The specific description of $\Gamma$ in each case is given in [24], but we shall not make use of this. The relevant information for the discussion below is the fact, shown by Tricerri [47], that all Inoue surfaces admit lcK metrics, except the surfaces of the type $S_{u}:=S_{N ; p, q, r ; u}^{+}$ for which the complex parameter $u$ is not real. In the latter case, Belgun [6] shows that there are no lcK metrics at all. Nevertheless, we proved in Theorem 1.1 that $S_{u}$ always admits timing locally conformally symplectic structures.

Let us now consider in a little more detail the analytic family $S_{u}, u \in \mathbb{C} \backslash \mathbb{R}$ of Inoue surfaces of the third type. We will show, by using an argument from [6], that in this case $\mathcal{T}\left(S_{u}\right)$ is a single point.

It is known $[6$, p. 35; 22, Theorem $1 ; 49]$ that $S_{u}=(\mathbb{C} \times \mathbb{H}) / \Gamma_{u}$ where $\Gamma_{u}$ is a lattice in the solvable Lie group

$$
\mathrm{Sol}_{1}^{\prime 4}=\left\{\left(\begin{array}{ccc}
1 & a & b+i \log \gamma \\
0 & \gamma & c \\
0 & 0 & 1
\end{array}\right), \gamma>0, a, b, c \in \mathbb{R}\right\} \subset \mathrm{GL}(3, \mathbb{C}) .
$$

The group $\mathrm{Sol}_{1}^{\prime 4}$ acts itself simply transitively (and holomorphically) on $\mathbb{C} \times \mathbb{H}$. This allows one to identify $S_{u}$ with the quotient $\mathrm{Sol}_{1}^{\prime 4}$, endowed with a (fixed) left-invariant 
integrable almost complex structure $J$, by the left action of the lattice $\Gamma_{u}$. In explicit terms, let

$$
Y=\left(\begin{array}{lll}
0 & 1 & 0 \\
0 & 0 & 0 \\
0 & 0 & 0
\end{array}\right) \quad Z=\left(\begin{array}{lll}
0 & 0 & 1 \\
0 & 0 & 0 \\
0 & 0 & 0
\end{array}\right) \quad T=\left(\begin{array}{lll}
0 & 0 & i \\
0 & 1 & 0 \\
0 & 0 & 0
\end{array}\right) \quad U=\left(\begin{array}{lll}
0 & 0 & 0 \\
0 & 0 & 1 \\
0 & 0 & 0
\end{array}\right)
$$

be the generators of the Lie algebra of $\mathrm{Sol}_{1}^{\prime 4}$ with

$$
Z \text { central, }[Y, T]=Y,[T, U]=U,[Y, U]=Z
$$

and denote with the same letters the induced left-invariant vector fields on $\mathrm{Sol}_{1}^{\prime 4}$. Then the left-invariant complex structure $J$ on $\mathrm{Sol}_{1}^{\prime 4}$ is given by [6, (35)]

$$
J Y=-Z, J Z=Y, J T=-U-Z, J U=T-Y .
$$

Furthermore, $\mathrm{Sol}_{1}^{4}$ admits an ad-invariant 1 -form $\alpha_{0}$, defined by $\alpha_{0}(T)=1, \alpha_{0}(U)=$ $\alpha_{0}(Y)=\alpha_{0}(Z)=0$, which descends to define a closed but not exact 1-form (still denoted by $\left.\alpha_{0}\right)$ on $S_{u}$. As $b_{1}\left(S_{u}\right)=1$, by Remark 2.3 we can assume without loss that $S_{u}$ admits a locally conformally symplectic 2-form $F$ which tames $J$, and whose Lee form equals $k \alpha_{0}$ for a non-zero real constant $k$. As $\operatorname{Sol}_{1}^{4}$ has a bi-invariant volume form $v$ [20, Lemma 4] (which defines a volume form on the quotient $S_{u}$, still denoted by $v$ ), for any left-invariant vector fields $U$ and $V$ on $\mathrm{Sol}_{1}^{4}$ (which define vector fields on the quotient $S_{u}$, still denoted by $U$ and $V$ ), one can consider the average of $F$ over $S_{u}$ :

$$
F_{0}(U, V):=\int_{S_{u}} F(U, V) v
$$

It can be shown, as in the proof of [6, Theorem 7], that $F_{0}$ defines a left-invariant 2-form on $\left(\mathrm{Sol}_{1}^{4}, J\right)$ which tames $J$ and satisfies $d F_{0}=k \alpha_{0} \wedge F_{0}$. Evaluating the later equality over $Y, Z, T$ yields (see also [6, (36)]) $F_{0}(Y, Z)=k F(Y, Z)$; as $Y=J Z$ and the $(1,1)$ part of $F_{0}$ is positive-definite, it follows that $k=1$. This shows that $\mathcal{T}\left(S_{u}\right)=\left\{a_{0}\right\}$ with $a_{0}=\left[\alpha_{0}\right]$. It is easy to check (see [20, Sect. 7.1]), using the explicit description of $S_{u}$ of [24] and the isomorphism $\mathbb{C}^{*}=H^{1}\left(S, \mathbb{C}^{*}\right) \cong \operatorname{Pic}_{0}(S)$ of [28, I, p. 756], that the corresponding holomorphic line bundle $\mathcal{L}_{a_{0}}$ is isomorphic to the anticanonical line bundle $\mathcal{K}_{S_{u}}^{*}$.

Noting finally that the existence of a locally conformally symplectic structure taming $J$ is an open condition (with respect to $J$ ) and that the Inoue surfaces of the type $S_{N, p, q, r, S}^{-}$are quotients of Inoue surfaces of the type $S_{N, p, q, r ; 0}^{+}$by an involution [24], we obtain the following 
Proposition 5.2. Let $S$ be an Inoue surface with $b_{2}(S)=0$ in one of the types $S_{N, p, q, r ; u}^{+}$or $S_{N ; p, q, r}^{-}$. Then $\mathcal{C}(S)$ and $\mathcal{T}(S)$ are given as follows:

- $\mathcal{C}(S)=\mathcal{T}(S)=\left\{a_{0}\right\}$ iff $S$ is of the type $S_{N ; p, q, r}^{-}$or $S_{N, p, q, r ; u}^{+}$with $u \in \mathbb{R}$;

- $\mathcal{C}(S)=\emptyset, \mathcal{T}(S)=\left\{a_{0}\right\}$ iff $S$ is of the type $S_{N, p, q, r ; u}^{+}$with $u \in \mathbb{C} \backslash \mathbb{R}$,

where $\mathcal{L}_{a_{0}}=\mathcal{K}_{S_{u}}^{*}$.

\subsection{Kato surfaces}

These are minimal complex surfaces in the Kodaira class VII whose second Betti number is strictly greater than 0 , and which have a global spherical shell (GSS). Conjecturally, any minimal surface in the class VII should be either a Hopf surface, an Inoue surface, or a Kato surface. However, this conjecture is still far from being solved.

For Kato complex surfaces, Brunella $[9,10]$ has shown that $\mathcal{C}(S) \neq \emptyset$ and that $\mathcal{C}(S)$ has $-\infty$ as an accumulation point. Note that any Kato surface $S$ is diffeomorphic to $\left(S^{1} \times S^{3}\right) \sharp k \overline{\mathbb{C} P^{2}}$ (where $k:=b_{2}(S)$ ); see for example, [34]. As $S^{1} \times S^{3}$, with a complex structure of a Hopf surface of class 1, admits a Vaisman metric, it follows by [32, 37] that $H_{d_{L}}^{3}\left(S^{1} \times S^{3}, L\right)=\{0\}$ for any non-trivial flat real line bundle $L$. By Lemma 3.3, $H_{d_{L}}^{3}(S, L)=\{0\}$ and, therefore, by Remark 3.2, $\mathcal{T}(S)$ must be an open subset of $(-\infty, 0)$. Similar conclusion holds true for $\mathcal{C}(S) \subseteq \mathcal{T}(S)$, by [20] and Lemma 2.10, together with the vanishing of $H^{2}(S, \mathcal{L})$ and $H^{0}(S, \mathcal{L})$ established in Lemmas 2.13 and 2.4 and 2.11, respectively.

Further progress in this case seems to depend on a better understanding of the subset $\mathcal{G}(S)$, as the following result suggests.

Proposition 5.3. Let $S$ be a Kato surface. Then,

$$
\mathcal{G}(S) \subseteq \mathcal{T}(S)
$$

Proof. Let $c \in \mathcal{G}(S)$. Brunella shows (see [9, Remark 9]) that there exists an lcK metric whose Lee form defines a de Rham class $b$ with $b<c$. By Proposition 4.4 and Lemma 2.13, any $a \in[b, c]$ (in particular $a=c$ ) belongs to $\mathcal{T}(S)$.

\section{Funding}

V.A. was supported in part by an NSERC Discovery Grant; V.A. and G.D. were supported in part by the ANT Grant MNGNK-ANR-10-BLAN-0118. 


\section{Acknowledgments}

V.A. is grateful to the Université Aix-Marseille and the Institute of Mathematics and Informatics of the Bulgarian Academy of Sciences where a part of this project was realized. G.D. is grateful to the UQAM for their hospitality during the preparation of the work. The authors are very grateful to Dimiter Vassilev for his invaluable help with the spectral theory of strongly elliptic operators, and to Dimitry Jakobson, François Hamel, and Fréderic Rochon for sharing with us their expertise on spectral analysis and the theory of elliptic PDE's. The authors are also grateful to Liviu Ornea and Misha Verbitsky for clarifying their results and giving them an access to the unpublished work [39]. They also thank the referee for her/his valuable suggestions on improving the presentation.

\section{Appendix A Perron-Type Theorem for Second-Order Strongly Elliptic Linear Operators on a Compact Riemannian Manifold}

We review here some spectral properties of the second-order strongly elliptic linear operators in the form

$$
L(u)=\Delta^{g} u+g(\alpha, d u)+c u
$$

where $g$ is a Riemannian metric on a compact manifold $M, \Delta^{g}=\delta^{g} d$ is the corresponding Riemannian Laplacian (which we will consequently denote by $\Delta$ ), $\alpha$ is a given smooth 1 -form and $c$ a given smooth function on $M$. Note that $L$ need not to be self-adjoint in general, a case where the spectral theory is well-established.

In a local chart $U, L$ takes the form

$$
L_{U}=-\sum_{i, j=1}^{n} a_{U}^{i j}(x) \frac{\partial^{2}}{\partial x_{i} \partial x_{j}}+\sum_{k=1} b_{U}^{k}(x) \frac{\partial}{\partial x_{k}}+c_{U}(x),
$$

where $a_{U}^{i j}, b_{U}^{k}, c_{U}$ are smooth real functions such that the symmetric matrix $\left(a_{U}^{i j}\right)$ is uniformly positive-definite on $U$. In this case, the Perron-type Theorem established in [16, p. 360; 35] states that if $\Omega \subset U$ is compactly supported domain with smooth boundary, the operator $L_{U}$ taken on smooth functions on $\bar{\Omega}$ with zero boundary value has real eigenvalue $\lambda_{0}$ (called principal eigenvalue) such that, for any other eigenvalue $\lambda$, $\operatorname{Re}(\lambda) \geq \lambda_{0}$; furthermore, $\lambda_{0}$ is simple and the corresponding eigenspace is generated by a nowhere vanishing smooth function $u_{0}$ on $\Omega$.

Recall that, for any strongly elliptic linear operator $L: \mathcal{C}^{\infty}(M) \rightarrow \mathcal{C}^{\infty}(M)$, the set of (complex) eigenvalues is discrete, having a limit point only at infinity; see for example, [7, p. 465] or [4, p. 126]. We want to establish the following adaptation of 
the Perron-type Theorem mentioned above to the case of a compact manifold without boundary (as we failed to find a reference to this result in the literature).

Theorem A.1. Let $L(u)=\Delta u+g(\alpha, d u)+c u$ be a linear strongly elliptic linear differential operator of order 2 on a compact Riemannian manifold $(M, g)$. Then, there exists a real eigenvalue $\lambda_{0}$ for $L$ which admits a smooth everywhere positive eigenfunction $u_{0}$. Furthermore, $\lambda_{0}$ satisfies the following properties:

(i) $\lambda_{0}$ is of multiplicity one.

(ii) If $\lambda$ is another eigenvalue, then $\operatorname{Re}(\lambda)>\lambda_{0}$.

(iii) $\lambda_{0}=\sup _{u \in \mathcal{A}}\left(\inf _{x \in M} \frac{L(u)}{u}\right)$, where $\mathcal{A}=\left\{u \in \mathcal{C}^{\infty}(M) \mid u>0\right\}$.

Definition A.2. The eigenvalue $\lambda_{0}$ is called the principal eigenvalue of $L$.

Proof of Theorem A.1. The proof will be divided into four steps, corresponding to the statements in the Theorem A.1 as follows.

Step 1. We shall establish in this Step the existence of a real eigenvalue $\lambda_{0}$ corresponding to an everywhere positive smooth eigenfunction $u_{0}$. To this end, we shall work with the Sobolov spaces $W_{k}^{2}(M)$ corresponding to the norm

$$
\|f\|_{k}:=\left[\sum_{0 \leq j \leq k} \int_{M}\left|D^{j} f\right|^{2} v_{g}\right]^{1 / 2},
$$

where $\left|D^{j} f\right|$ is the point-wise norm of the $j$ th covariant derivative and $v_{g}$ is the Riemannian volume form. In what follows, we shall choose $k$ sufficiently large so that we have a continuous embedding $W_{k}^{2}(M) \subset \mathcal{C}^{2}(M)$. Because $M$ is compact, we also have the continuous embeddings

$$
W_{k}^{2}(M) \subset W_{l}^{2}(M)
$$

for any integers $0 \leq l \leq k$.

We shall assume (without loss) in what follows that the smooth function $c \geq 0$ (otherwise we consider the operator $L-\left(\inf _{M} C\right)$ Id instead of $L$ ). Then, by the maximum principle [4, III, Section 8, 3.71] (which in the sequel we shall always apply to $-L$ and $-u$ ), 0 is not an eigenvalue of $L$, that is, $\operatorname{Ker} L=\{0\}$. It is a standard fact that $L: W_{k+2}^{2}(M) \rightarrow W_{k}^{2}(M)$ is then invertible, and $L^{-1}: W_{k}^{2}(M) \rightarrow W_{k+2}^{2}(M)$ is bounded. Indeed, as $L=\Delta+T$ with $\Delta$ being the self-adjoint Riemannian Laplacian and $T$ of order $\leq 1$, the composition $T: W_{k+2}^{2}(M) \rightarrow W_{k+1}^{2}(M) \subset W_{k}^{2}(M)$ is a compact operator by the Rellich-Kondrachov theorem (see [7, p. 458]); it follows that Index $(L)=\operatorname{Index}(\Delta)=0$ 
by [23, Cor. 19.1.8] and, as $\operatorname{Ker} L=\{0\}, L$ is invertible and bounded (by the standard $L^{p}$-estimates, see e.g. [7, p. 463]). Using the compactness of $W_{k+1}^{2}(M) \subset W_{k}^{2}(M)$ again, we conclude that the composition

$$
A: W_{k}^{2}(M) \stackrel{L^{-1}}{\longrightarrow} W_{k+2}^{2} \subset W_{k}^{2}(M)
$$

is a compact operator. We define the cone

$$
C:=\left\{u \in W_{k}^{2}(M) \mid u \geq 0\right\}
$$

By the maximum principle [4, III, Section 8, 3.71] again, we have that $A(C) \subset C$ and if $w \in C$ with $w \neq \equiv$, then $A w>0$. Using standard elliptic regularity (see e.g. [7, p. 467]), it will be enough to show that $A$ has a non-trivial eigenfunction $w_{0} \in C$, corresponding to a real positive eigenvalue $1 / \lambda_{0}$ (we will have then that $u_{0}:=\lambda_{0} A\left(w_{0}\right)$ is a smooth strictly positive eigenfunction of $L$, corresponding to the real positive eigenvalue $\lambda_{0}$ ).

Let us fix a function $w \in C, w \neq \equiv$, and let $v:=A w$. By continuity, there exists $\mu>0$ such that

$$
\mu v \geq w
$$

on $M$. We claim that if the equation

$$
u=\lambda A(u+\epsilon w)
$$

with $\epsilon>0$ and $\lambda>0$ has an everywhere positive solution $u$, then necessarily

$$
\lambda \leq \mu \text {. }
$$

Indeed,

$$
u=\lambda A(u)+\lambda A(\epsilon w)>\lambda A(\epsilon w)=\lambda \epsilon v \geq\left(\frac{\lambda}{\mu}\right) \epsilon w
$$

hence

$$
u \geq \lambda A(u) \geq \lambda A\left(\frac{\lambda \epsilon}{\mu} w\right)=\frac{\lambda^{2}}{\mu} \epsilon v \geq\left(\frac{\lambda}{\mu}\right)^{2} \epsilon w .
$$

By induction, for $k \geq 1$,

$$
u \geq\left(\frac{\lambda}{\mu}\right)^{k} \epsilon w
$$

which is possible only if $\lambda \leq \mu$.

We are now going to show that, for any $\epsilon>0$, the closed subset of $W_{k}^{2}(M)$

$$
S_{\epsilon}:=\{u \in C \mid \exists \lambda, \quad 0 \leq \lambda \leq 2 \mu, u=\lambda A(u+\epsilon w)\}
$$


is unbounded. To this end, we shall use (A.2) in conjunction with the well-known Schaefer theorem (see [16, p. 540]), which states that if $\tilde{A}: W \rightarrow W$ is a continuous compact (not necessarily linear) mapping of a Banach space $W$ and $C \subset W$ is a convex subset stable by $\tilde{A}$, then $\tilde{A}$ has a fixed point in $C$ provided that the subset

$$
\{u \in C \mid \exists \lambda, 0 \leq \lambda \leq 1 \text { such that } u=\lambda \tilde{A}(u)\}
$$

is bounded.

In our case, $W=W_{k}^{2}(M)$,

$$
\tilde{A}(u):=2 \mu(A(u)+\epsilon v)
$$

which is continuous compact because $A$ is, and $C \subset W_{k}^{2}(M)$ is the convex subset introduced in (A.1). Furthermore, if $S_{\epsilon}$ were bounded, $\tilde{A}$ would satisfy the hypothesis of the Schaefer theorem with respect to $C$, so $\tilde{A}$ would have a fixed point $u$ in $C$, that is, $u=\tilde{A} u=2 \mu A(u+\epsilon w)$, which contradicts (A.2).

Since $S_{\epsilon}$ is not bounded, there exists $u_{\epsilon} \in S_{\epsilon}$ with $\left\|u_{\epsilon}\right\|_{k} \geq \frac{1}{\epsilon}$. Denote by $\lambda_{\epsilon}\left(0 \leq \lambda_{\epsilon} \leq\right.$ $2 \mu$ ) the corresponding real number such that

$$
u_{\epsilon}=\lambda_{\epsilon} A\left(u_{\epsilon}+\epsilon w\right) \text {. }
$$

For any sequence $\epsilon_{m} \rightarrow 0$ let $\lambda_{m}:=\lambda_{\epsilon_{m}}$ and $u_{m}:=\frac{u_{\epsilon_{m}}}{\left\|u_{\epsilon_{m}}\right\|_{k}} \in C$, so that

$$
u_{m}=\lambda_{m} A\left(u_{m}+\epsilon_{m} \frac{w}{\left\|u_{\epsilon_{m}}\right\|_{k}}\right)
$$

Since $A$ is compact, we can take a subsequence if necessary and suppose that $\left(\lambda_{m}\right)$ and $\left(u_{m}\right)$ are convergent. Let $\lambda_{0}:=\lim _{m \rightarrow \infty} \lambda_{m}$ and $u_{0}:=\lim _{m \rightarrow \infty} u_{m} \geq 0$. Taking limit in (A.3), we get

$$
u_{0}=\lambda_{0} A\left(u_{0}\right)
$$

The condition $\left\|u_{m}\right\|_{k}=1$ prevents $\lambda_{0}$ and $u_{0}$ from vanishing. Thus, $u_{0}=\lambda_{0} A\left(u_{0}\right)>0$, and applying $L$ to the above equality, we obtain $L\left(u_{0}\right)=\lambda_{0} u_{0}$ with $\lambda_{0}>0$.

Step 2. We will now prove that the multiplicity of $\lambda_{0}$ is one.

As $L$ is a real operator, it is enough to consider a smooth non-identically zero real valued function $u$ on $M$, such that $L u=\lambda_{0} u$. Furthermore, replacing $u$ by $-u$ if necessary, we shall assume that $u$ is somewhere positive. Thus, letting

$$
\chi=\sup \left\{\mu>0 \mid u_{0}-\mu u \geq 0 \text { on } M\right\}
$$

we then have $\chi>0, v:=u_{0}-\chi u \geq 0$ on $M$, and hence $L v=\lambda_{0} v \geq 0$. 
By the Hopf maximum principle [4, III, Section 8, 3.71], we have that either $v>0$ on $M$ or $v \equiv 0$. By the definition of $\chi$ we conclude that $v \equiv 0$.

Step 3. We now show that, for any other (complex) eigenvalue $\lambda$ of $L, \operatorname{Re}(\lambda)>\lambda_{0}$.

Let $u \neq 0$ be a complex-valued smooth function on $M$ with $L u=\lambda u$ and let $v:=u / u_{0}$. We then have

$$
\begin{aligned}
\lambda u_{0} v=L\left(u_{0} v\right) & =v L\left(u_{0}\right)+u_{0} L(v)-c u_{0} v-2 g\left(d u_{0}, d v\right) \\
& =\lambda_{0} u_{0} v+u_{0}\left(\Delta v+g\left(\alpha-2 d \log u_{0}, d v\right)\right)=\lambda_{0} u_{0} v+u_{0} K(v),
\end{aligned}
$$

where we have set

$$
K(v):=\Delta(v)+g\left(\alpha-2 d \log u_{0}, d v\right) .
$$

Dividing the last equality by $u_{0}$, we have

$$
K(v)=\left(\lambda-\lambda_{0}\right) v,
$$

and, as $K$ is a real operator,

$$
K(\bar{v})=\left(\bar{\lambda}-\lambda_{0}\right) \bar{v} .
$$

It follows

$$
\begin{aligned}
K\left(|v|^{2}\right)=K(v \bar{v}) & =v K(\bar{v})+\bar{v} K(v)-2 g(d v, d \bar{v}) \\
& =2\left(\operatorname{Re}(\lambda)-\lambda_{0}\right)|v|^{2}-2 g(d v, d \bar{v}) \leq 2\left(\operatorname{Re}(\lambda)-\lambda_{0}\right)|v|^{2}
\end{aligned}
$$

Suppose for a contradiction that $\operatorname{Re}(\lambda)-\lambda_{0} \leq 0$. Then, the Hopf maximum principle [4, III, Section 8, 3.71] implies $|v|=$ const, and therefore

$$
0=K\left(|v|^{2}\right) \leq 2\left(\operatorname{Re}(\lambda)-\lambda_{0}\right)|v|^{2}
$$

showing that $\operatorname{Re}(\lambda)=\lambda_{0}$ (as $\left.v=u / u_{0} \not \equiv 0\right)$. Going back to (A.4) (and using again $K\left(|v|^{2}\right)=0$ ), we have

$$
0=K\left(|v|^{2}\right)=-2 g(d v, d \bar{v})+2\left(\operatorname{Re}(\lambda)-\lambda_{0}\right)|v|^{2}=-2\|d v\|_{g}^{2},
$$

that is, $v=u / u_{0}$ is a constant. As $\lambda \neq \lambda_{0}$ by assumption, this is a contradiction. Step 4. We finally have to prove that

$$
\lambda_{0}=\sup _{u \in \mathcal{A}} \inf _{x \in M} \frac{L(u)}{u}
$$

where $\mathcal{A}=\left\{u \in \mathcal{C}^{\infty}(M) \mid u>0\right\}$. 
To this end, we will show first that the formal conjugate operator

$$
L^{*}(u)=\Delta u-g(\alpha, d u)+\left(\delta^{g} \alpha+c\right) u
$$

of $L$ (with respect to the global $L_{2}$ product on $(M, g)$ ) has the same principal eigenvalue as $L$. Indeed, let $\lambda_{0}^{*}$ be the principal eigenvalue of $L^{*}$ and $u_{0}^{*}>0$ be an eigenfunction. Then

$$
\lambda_{0} \int_{M} u_{0} u_{0}^{*} v_{g}=\int_{M} L\left(u_{0}\right) u_{0}^{*} v_{g}=\int_{M} u_{0} L^{*}\left(u_{0}^{*}\right) v_{g}=\lambda_{0}^{*} \int_{M} u_{0} u_{0}^{*} v_{g} .
$$

Since $u_{0}>0$ and $u_{0}^{*}>0$, it follows that $\lambda_{0}=\lambda_{0}^{*}$.

Now, let

$$
\mu:=\sup _{u \in \mathcal{A}} \inf _{x \in M} \frac{L(u)}{u}
$$

We clearly have $\mu \geq \inf _{x \in M} \frac{L\left(u_{0}\right)}{u_{0}}=\lambda_{0}$. To establish the converse inequality, let ( $\left.w_{m}\right)$ be a maximizing sequence in $\mathcal{A}$ such that

$$
\mu-\frac{1}{m} \leq \inf _{x \in M} \frac{L\left(w_{m}\right)}{w_{m}} \leq \mu .
$$

It then follows

$$
\lambda_{0} \int_{M} w_{m} u_{0}^{*} \quad v_{g}=\int_{M} w_{m} L^{*}\left(u_{0}^{*}\right) \quad v_{g}=\int_{M} L\left(w_{m}\right) u_{0}^{*} \quad v_{g} \geq\left(\mu-\frac{1}{m}\right) \int_{M} w_{n} u_{0}^{*} v_{g},
$$

thus showing $\lambda_{0} \geq \mu$.

The following result follows from the general Rellich-Kato theory, see for example, [26, II, Section 1.8; IV, Theorem 3.16, and VII, Theorem 1.7] applied to the bounded operator $A$ constructed in the proof of Theorem A.1, by noting that the principal eigenvalue $\lambda_{0}$ of $L$ is simple and can be separated from the remainder of the spectrum.

Theorem A.3. Let $L(t)$ be an analytic family of linear strongly elliptic operators as in Theorem A.1. For each $t$, denote by $\lambda_{0}(t)$ the principal eigenvalue of $L(t)$ with corresponding eigenfunction $u_{t}>0$, normalized by $\int_{M} u_{t}^{2} v_{g}=1$. Then $\lambda_{0}(t)$ and $u_{t}$ vary analytically with respect to $t$.

\section{References}

[1] Apostolov, V., M. Bailey, and G. Dloussky. "From locally conformally Kähler to bihermitian structures on non-Kähler complex surfaces." Mathematical Research Letters 22, no. 2 (2015): 317-36. 
[2] Apostolov, V., P. Gauduchon, and G. Grantcharov. "Bihermitian structures on complex surfaces." Proceedings of the London Mathematical Society (3) 79, no. 2 (1999): 414-28. Corrigendum 92, no. 1 (2006): 200-02.

[3] Apostolov, V. and M. Gualtieri. “Generalized Kähler manifolds, commuting complex structures and split tangent bundles." Communications in Mathematical Physics 271, no. 2 (2007): 561-75.

[4] Aubin, Th. Some Nonlinear Problems in Riemannian Geometry. Springer Monographs. 1998.

[5] Barth, W., K. Hulek, C. Peters, and A. VandeVen. Compact Complex Surfaces, 2nd ed. Heidelberg: Springer, 2004.

[6] Belgun, F. "On the metric structure of non-Kähler complex surfaces." Mathematische Annalen 317, no. 1 (2000): 1-40.

[7] Besse, A. L. Einstein Manifolds. Ergeb. Math. Grenzgeb. 3. Heidelberg, New York: Springer, Berlin, 1987.

[8] Bogomolov, F. “Classification of surfaces of class $\mathrm{VII}_{0}$ with $b_{2}=0$." Mathematics of the USSR-Izvestiya 10, no. 2 (1976): 255-69.

[9] Brunella, M. "Locally conformally Kähler metrics on certain non-Kählerian surfaces." Mathematische Annalen 346, no. 3 (2010): 629-39.

[10] Brunella, M. "Locally conformally Kähler metrics on Kato surfaces." Nagoya Mathematical Journal 202 (2011): 77-81.

[11] Buchdahl, N. “On compact Kähler surfaces." Annales de l'institut Fourier 49, no. 1 (1999): 287-302.

[12] Buchdahl, N. "A Nakai-Moishezon criterion for non-Kähler surfaces." Annales de l'institut Fourier 50, no. 5 (2000): 1533-8.

[13] Demailly, J.-P. and M. Paun. "Numerical characterization of the Kähler cone of a compact Kähler manifold." Annals of Mathematics 159, no. 3 (2004): 1247-74.

[14] Dragomir, S. and L. Ornea. Locally Coformal Kähler Geometry. Progress in Mathematics 155. Boston, MA: Birkhäuser Boston, 1998.

[15] Donaldson, S. K. "Two-Forms on Four-Manifolds and Elliptic Equations." In Inspired by S. S. Chern, 153-72. Nankai Tracts Math. 11. Hackensack, NJ: World Science Publisher, 2006.

[16] Evans, L. C. Partial Differential Equations. 2nd ed. Graduate Studies in Mathematics 19. Providence: AMS, 2010.

[17] Gauduchon, P. "Le théorème d'excentricité nulle." Comptes Rendus de l'Acadmie des Sciences 285, no. 5 (1977): 387-90.

[18] Gauduchon, P. "La 1-forme de torsion d'une variété hermitienne." Mathematische Annalen 267, no. 4 (1984): 495-518.

[19] Gauduchon, P. and L. Ornea. "Locally conformally Kähler metrics on Hopf surfaces." Annales de l'Institut Fourier 48, no. 4 (1998): 1107-27.

[20] Goto, R. "On the stability of locally conformal Kähler structures." (2010): preprint arxiv: $1012.2285 \mathrm{v} 1$. 
[21] Harvey, R. and H. Blaine Lawson, Jr. "An intrinsic characterization of Kähler manifolds." Inventiones Mathematicae 74, no. 2 (1983): 169-98.

[22] Hasegawa, K. "Complex and Kähler structures on compact solvmanifolds." Journal of Symplectic Geometry 3, no. 4 (2005): 749-67.

[23] Hormander, L. The Analysis of Linear Partial Differential Operators III. Heidelberg, Berlin, New York: Springer, 1994.

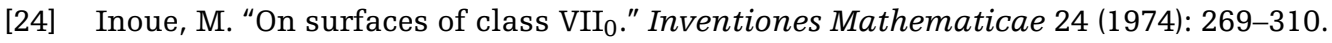

[25] Kato, M. "Topology of Hopf surfaces." Journal of the Mathematical Society of Japan 27 (1975): 222-38. Erratum Journal of the Mathematical Society of Japan 41 (1989): 173-74.

[26] Kato, T. Perturbation Theory for Linear Operators. Heidelberg, Berlin, New York: Springer, 1980.

[27] Kobayashi, S. and K. Nomizu. Foundations of Differential Geometry, vol. II, 179. New York: Interscience Publishers, 1963.

[28] Kodaira, K. "On the structure of complex analytic surfaces I, II, III." American Journal of Mathematics 86 (1966): 751-98; American Journal of Mathematics 88 (1966): 682-721; American Journal of Mathematics 90 (1968): 55-83.

[29] Kokarev, G. "On pseudo-harmonic maps in conformal geometry." Proceedings of the London Mathematical Society 99, no. 1 (2009): 168-94.

[30] Lamari, A. “Courants kählériens et surfaces compactes." Annales de l'institut Fourier 49, no. 1 (1999): 263-85.

[31] Lamari, A. "Le cone kählérien dune surface." Journal des Mathématiques Pures et Appliquées 78, no. 3 (1999): 249-63.

[32] de Léon, M., B. López, J. C. Marrero, and E. Padrón. “On the computation of the Lichnerowicz-Jacobi cohomology." Journal of Geometry and Physics 44, no. 4 (2003): 507-22.

[33] Li, J. and S. T. Yau. "Hermitian Yang-Mills Connections on non-Kähler Manifolds." Mathematical Aspects of String Theory (San Diego, CA, 1986), 560-73. Advanced Series in Mathematical Physics 1. Singapore: World Scientific Publishing, 1987.

[34] Nakamura, I. "On surfaces of class $V I I_{0}$ with curves." Inventiones Mathematicae 78, no. 3 (1984): 393-43.

[35] Ni, L. "A Perron-type theorem on the principal eigenvalue of nonsymmetric elliptic operator." American Mathematical Monthly 121, no. 10 (2014): 903-8.

[36] Ornea, L. and M. Verbitsky. “Topology of Locally Conformally Kähler Manifolds with Potential." International Mathematics Research Notices 2010, no. 4 (2009): 717-26.

[37] Ornea, L. and M. Verbitsky. "Morse-Novikov cohomology of locally conformally Kähler manifolds." Journal of Geometry and Physics 59, no. 3 (2009): 295-305.

[38] Ornea, L. and M. Verbitsky. A report on Locally Conformally Kähler Manifolds. Harmonic Maps and Differential Geometry. 135-49. Contemporary Mathematics 542. Providence, RI: American Mathematical Society, 2011. 
[39] Ornea, L. and M. Verbitsky. "Compact pluricanonical manifolds are Vaisman." in preparation.

[40] Pontecorvo, M. “Complex structures on Riemannian 4-manifolds." Mathematische Annalen 309, no. 1 (1997): 159-77.

[41] Rellich, F. Perturbation Theory of Eigenvalue Problems. New York, Gordon and Breach: 1969.

[42] Siu, Y.-T. "Every $K 3$ surface is Kähler." Inventiones Mathematicae 73, no. 1 (1983): 139-50.

[43] Taubes, C. H. "Tamed to compatible: symplectic forms via moduli space integration." Journal of Symplectic Geometry 9, no. 2 (2011): 161-250.

[44] Teleman, A. "Projectively flat surfaces and Bogomolov's theorem on class $\mathrm{VII}_{0}$-surfaces." International Journal of Mathematics 5, no. 2 (1994): 253-64.

[45] Teleman, A. "The pseudo-effective cone of a non-Kählerian surface and applications." Mathematische Annalen 335 (2006): 965-89.

[46] Todorov, A. "Applications of the Kähler-Einstein-Calabi-Yau metric to moduli of $K 3$ surfaces." Inventiones Mathematicae 61, no. 3 (1980): 251-65.

[47] Tricerri, F. "Some examples of locally conformal Kähler manifolds." Rendiconti del Seminario Matematico Universitàe Politecnico di Torino 40, no. 1 (1982): 81-92.

[48] Vuletescu, V. "Blowing-up points on locally conformally Kähler manifolds." Bulletin Mathematique de la Societe des Sciences Mathematiques de Roumanie 52, no. 3 (2009): 387-90.

[49] Wall, C. I. C. "Geometric structures on compact complex analytic surfaces." Topology 25, no. 2 (1986): 119-53. 